\title{
Noncommutative Relativistic Spacetimes and Worldlines from 2 + 1 Quantum (Anti-)de Sitter Groups
}

\author{
Ángel Ballesteros, N. Rossano Bruno, and Francisco J. Herranz \\ Departamento de Física, Universidad de Burgos, 09001 Burgos, Spain \\ Correspondence should be addressed to Francisco J. Herranz; fherranz@ubu.es
}

Received 12 May 2017; Accepted 6 August 2017; Published 28 November 2017

Academic Editor: Edward Sarkisyan-Grinbaum

Copyright (C) 2017 Ángel Ballesteros et al. This is an open access article distributed under the Creative Commons Attribution License, which permits unrestricted use, distribution, and reproduction in any medium, provided the original work is properly cited. The publication of this article was funded by $\mathrm{SCOAP}^{3}$.

\begin{abstract}
The $\kappa$-deformation of the $(2+1) \mathrm{D}$ anti-de Sitter, Poincaré, and de Sitter groups is presented through a unified approach in which the curvature of the spacetime (or the cosmological constant) is considered as an explicit parameter. The Drinfel'd-double and the Poisson-Lie structure underlying the $\kappa$-deformation are explicitly given, and the three quantum kinematical groups are obtained as quantizations of such Poisson-Lie algebras. As a consequence, the noncommutative $(2+1) \mathrm{D}$ spacetimes that generalize the $\kappa$-Minkowski space to the (anti-)de Sitter ones are obtained. Moreover, noncommutative 4D spaces of (time-like) geodesics can be defined, and they can be interpreted as a novel possibility to introduce noncommutative worldlines. Furthermore, quantum (anti-)de Sitter algebras are presented both in the known basis related to $2+1$ quantum gravity and in a new one which generalizes the bicrossproduct one. In this framework, the quantum deformation parameter is related to the Planck length, and the existence of a kind of "duality" between the cosmological constant and the Planck scale is also envisaged.
\end{abstract}

\section{Introduction}

The connection between quantum groups and Planck scale physics was early suggested in [1]. Quantum deformations of Lie algebras and Lie groups [2-8] have been broadly applied in the construction of deformed symmetries of spacetimes [9-23], especially for the Poincaré and Galilei cases, for which the deformation parameter is known to play the role of a fundamental scale. Among all these quantum kinematical algebras the well known $\kappa$-Poincaré algebra $[9,13,14,16,18]$ has been frequently considered.

These deformed Poincaré symmetries were later applied in the context of the so-called doubly special relativity (DSR) theories [24-32] which introduced two fundamental scales: the usual observer-independent velocity scale $c$ as well as an observer-independent length scale $l_{p}$, which was related to the deformation parameter in the algebra. Since from all approaches to quantum gravity [33-37] the Planck scale is thought to play a fundamental role, DSR theories seem to establish a promising link between some Planck scale effects and quantum groups $[38,39]$.

From a more general viewpoint, we recall that noncommutative spaces have been proposed as a suitable algebraic framework in order to describe the "quantum" structure of the geometry of spacetime at the Planck scale through a noncommutative algebra of quantum spacetime coordinates [4044]. In this way the deformation parameter characterizes the noncommutativity of the spacetime algebra, thus generating uncertainty relations between noncommuting coordinates that can be thought to model a "fuzzy" or "discrete" nature of the spacetime at very small distances (or high energies) [45, 46]. In particular, the noncommutative spacetime deduced from the $\kappa$-Poincaré algebra is the so-called $\kappa$-Minkowski spacetime $[15,22]$, which is the algebra defined by the spacetime quantum group coordinates dual to the translation (momenta) generators.

In this framework, spacetime curvature (or nonzero cosmological constant) should play a relevant role concerning 
the possible cosmological consequences of a quantum spacetime (see, e.g., [38, 47-51] and references therein). Therefore, it seems natural to consider the construction of the $\kappa$ deformation for the (anti-)de Sitter (hereafter (A)dS) groups, and to analyse their possible connections with quantum gravity theories with a nonzero cosmological constant. In this respect, we recall that the Hopf structure for the $\kappa$ deformation of $(2+1) \mathrm{D}(\mathrm{A}) \mathrm{dS}$ and Poincaré $(\mathscr{P})$ algebras were collectively obtained in [19], and their connection between their deformed commutation rules and $2+1$ quantum gravity has been explored in [38]. The results obtained in [19] correspond to the l.h.s. of the commutative diagram:

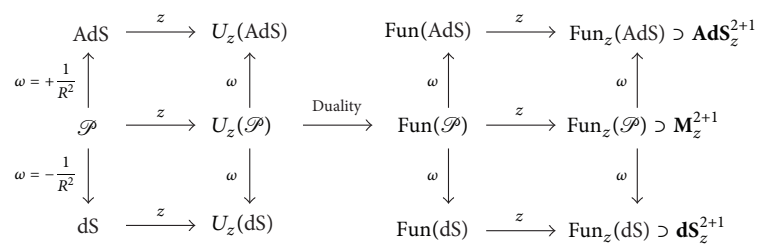

where vertical arrows indicate a classical deformation [52, 53] that introduces the spacetime curvature $\omega$ (or cosmological constant $\Lambda=-\omega$ ) related to the (A)dS radius $R$ by $\omega= \pm 1 / R^{2}$, and the horizontal ones show the quantum deformation with parameter $z=1 / \kappa$ (related to the Planck length $l_{p}$ ); reversed arrows correspond to the spacetime contraction $\omega \rightarrow 0$ and (classical) nondeformed limit $z \rightarrow 0$. As a consequence, the construction of noncommutative (A)dS spacetimes in terms of intrinsic and ambient spacetime quantum group coordinates seems worth being explored in detail and, moreover, the same framework could account for new proposals of noncommutative spaces of time-like geodesics (worldlines), which, to the best of our knowledge, have not been considered in the literature yet, even for the Poincaré case.

Here we present an enlarged and updated review version of our unpublished manuscript arXiv:hep-th/0401244, in which the above-mentioned problems are faced for the three relativistic cases simultaneously, that is, by dealing explicitly with the spacetime curvature $\omega$ as a contraction parameter. Hence, we propose to explore the r.h.s. of the diagram (1) (dual to the 1.h.s.) by computing the quantum deformation of the $(2+1) \mathrm{D}(\mathrm{A}) \mathrm{dS}$ groups (that is, $\operatorname{Fun}_{z}((\mathrm{~A}) \mathrm{dS})$ ) which are obtained by quantizing the Poisson-Lie algebra of smooth functions on these groups (namely, Fun((A)dS)) coming from a suitable classical $r$-matrix. In this way, the $(2+1) \mathrm{D}$ noncommutative spaces (e.g., $\left.\mathbf{A d S}_{z}^{2+1}\right)$ can then be identified as certain subalgebras of the corresponding quantum groups. Moreover, we also construct and study in detail the corresponding $4 \mathrm{D}$ noncommutative spaces of worldlines. We stress that in our approach all the $\kappa$ Poincaré relations (including its noncommutative spaces) can be directly recovered from the general (A)dS expressions through the limit $\omega \rightarrow 0$. Moreover, all of the resulting noncommutative spaces are covariant under quantum group (co)actions (for the construction of Poisson and quantum homogeneous spaces we refer to [54-58] and references therein).
The structure of the paper is the following. In the next section we recall the basics on the (A)dS groups in $(2+$ 1) dimensions and their associated homogeneous $(2+1) \mathrm{D}$ spacetimes and 4D spaces of worldlines (time-like lines). Both types of spaces are described in terms of intrinsic quantities (related to group parameters) as well as in ambient coordinates with one and two extra dimensions, respectively, which will be further used in their noncommutative versions. By starting from the classical $r$-matrix that generates the $\kappa$ deformation, we construct in Section 3 the corresponding Drinfel'd-double and obtain some preliminary information on the first-order quantum deformation, from which firstorder noncommutative spaces arise. On one hand, we find that at first-order in the deformation parameter the three noncommutative relativistic spacetimes are given by the same $\kappa$-Minkowski algebra. Moreover, we show that the deformation parameter can be interpreted as a curvature on a classical dS spacetime for the three cases, thus generalizing the results obtained in $[29,30]$ for $\kappa$-Poincaré. On the other hand, we obtain that the first-order noncommutative spaces of worldlines are in fact nondeformed ones, and a relationship with the nonrelativistic (Newtonian) kinematical groups is thus established.

As an intermediate stage in the search of the quantum (A)dS groups, we compute in Section 4 the invariant (A)dS vector fields and next the Poisson-Lie structures coming from the classical $r$-matrix generating the $\kappa$-deformation. These results enable us to propose in Section 5 the noncommutative $(\mathrm{A}) \mathrm{dS}$ spaces, which are written in both intrinsic and ambient coordinates. The resulting noncommutative spacetimes show how the curvature modifies the underlying firstorder $\kappa$-Minkowski space, while for the noncommutative spaces of worldlines we find that $2 \mathrm{D}$ velocity/rapidity space (spanned by the dual coordinates to the boost generators) remains nondeformed for $\kappa$-Poincaré but becomes deformed for the $(\mathrm{A}) \mathrm{dS}$ cases. Hence Lorentz invariance seems to be lost (or somewhat "deformed") when a nonzero curvature/cosmological constant is considered.

Section 6 is devoted to study the (dual) quantum (A)dS algebras and their deformed Casimirs in two different bases. In particular, starting from the expressions given in [19], a nonlinear transformation involving the generators of the stabilizer subgroup of a time-like line allows us to obtain these quantum algebras in a new basis that generalizes for any $\omega$ the bicrossproduct basis of $\kappa$-Poincaré [16]. These results are analysed in connection with $2+1$ quantum gravity [38] and a "duality" between curvature/cosmological constant and deformation parameter/Planck length is suggested along the same lines of the so-called "semidualization" approach for Hopf algebras in $2+1$ quantum gravity [59] associated with the exchange of the cosmological length scale and the Planck mass (see also [60, 61]). Finally, some remarks and comments concerning recent findings in this framework close the paper.

\section{2. (Anti-)de Sitter Lie Groups and Their Homogeneous Spaces}

The Lie algebras of the three $(2+1)$ D relativistic spacetimes of constant curvature can collectively be described by means of 
TABLE 1: The AdS, Minkowskian, and dS (2+1)D spacetimes and 4D spaces of time-like lines according to the curvature/cosmological constant $\omega=-\Lambda$.

\begin{tabular}{lcc}
\hline$\omega, \Lambda$ & Spacetime $\mathbb{S}_{(1)}$ with curvature $\omega$ & Space of worldlines $\mathbb{S}_{(2)}$ with curvature -1 \\
\hline$\omega>0, \Lambda<0$ & $\mathbf{A d S}^{2+1}=S O(2,2) / S O(2,1)$ & LAdS $^{2 \times 2}=S O(2,2) /(S O(2) \otimes S O(2))$ \\
$\omega=\Lambda=0$ & $\mathbf{M}^{2+1}=I S O(2,1) / S O(2,1)$ & $\mathbf{L M}^{2 \times 2}=I S O(2,1) /(S O(2) \otimes \mathbb{R})$ \\
$\omega<0, \Lambda>0$ & $\mathbf{d S}^{2+1}=S O(3,1) / S O(2,1)$ & $\mathbf{L d S}^{2 \times 2}=S O(3,1) /(S O(2) \otimes S O(1,1))$ \\
\hline
\end{tabular}

a real (graded) contraction parameter $\omega$ [19], and we denote them by $s_{\omega}(2,2)$. If $\left\{J, P_{0}, \mathbf{P}=\left(P_{1}, P_{2}\right), \mathbf{K}=\left(K_{1}, K_{2}\right)\right\}$ are, in this order, the generators of rotations, time translations, space translations, and boosts, the commutation relations of so $\omega(2,2) \mathrm{read}$

$$
\begin{gathered}
{\left[J, P_{i}\right]=\epsilon_{i j} P_{j},} \\
{\left[J, K_{i}\right]=\epsilon_{i j} K_{j},} \\
{\left[J, P_{0}\right]=0,} \\
{\left[P_{i}, K_{j}\right]=-\delta_{i j} P_{0},} \\
{\left[P_{0}, K_{i}\right]=-P_{i},} \\
{\left[K_{1}, K_{2}\right]=-J,} \\
{\left[P_{0}, P_{i}\right]=\omega K_{i},} \\
{\left[P_{1}, P_{2}\right]=-\omega J,}
\end{gathered}
$$

where from now on we assume that Latin indices $i, j=$ 1,2 , Greek ones $\mu, \nu=0,1,2, \hbar=c=1$, and $\epsilon_{i j}$ is a skew symmetric tensor such that $\epsilon_{12}=1$. For a positive, zero, and negative value of $\omega, s_{\omega}(2,2)$ provides a Lie algebra isomorphic to $s o(2,2)$, iso $(2,1)$, and $s o(3,1)$, respectively. The case $\omega=0$ can also be understood as an Inönü-Wigner contraction [62]: $s o(2,2) \rightarrow i s o(2,1) \leftarrow s o(3,1)$.

Parity $\Pi$ and time-reversal $\Theta$ are involutive automorphisms of $s o_{\omega}(2,2)$ defined by [63]

$$
\begin{aligned}
& \Pi:\left(P_{0}, \mathbf{P}, \mathbf{K}, J\right) \longrightarrow\left(P_{0},-\mathbf{P},-\mathbf{K}, J\right), \\
& \Theta:\left(P_{0}, \mathbf{P}, \mathbf{K}, J\right) \longrightarrow\left(-P_{0}, \mathbf{P},-\mathbf{K}, J\right),
\end{aligned}
$$

which together with the composition,

$$
\Pi \Theta:\left(P_{0}, \mathbf{P}, \mathbf{K}, J\right) \longrightarrow\left(-P_{0},-\mathbf{P}, \mathbf{K}, J\right)
$$

and the identity determine a $\mathbb{Z}_{2} \otimes \mathbb{Z}_{2} \otimes \mathbb{Z}_{2}$ Abelian group of involutions [64]. The automorphisms $\Pi \Theta$ and $\Pi$ give rise, in this order, to the Cartan decompositions:

$$
\begin{aligned}
& s_{\omega}(2,2)=h_{(1)} \oplus p_{(1)}, \\
& h_{(1)}=\langle\mathbf{K}, J\rangle \simeq s o(2,1), p_{(1)}=\left\langle P_{0}, \mathbf{P}\right\rangle, \\
& s o_{\omega}(2,2)=h_{(2)} \oplus p_{(2)}, \\
& h_{(2)}=\left\langle J, P_{0}\right\rangle \simeq s o(2) \oplus s o_{\omega}(2), p_{(2)}=\langle\mathbf{P}, \mathbf{K}\rangle,
\end{aligned}
$$

where $h_{(1)} \simeq s o(2,1)$ is the Lorentz subalgebra and $s o_{\omega}(2)=$ $\left\langle P_{0}\right\rangle$ covers the Lie subalgebras $s o(2)$, iso $(1) \simeq \mathbb{R}$, and $s o(1,1)$ for $\omega>,=,<0$, correspondingly. In fact, the contraction parameter $\omega$ is related to the $\mathbb{Z}_{2}$-grading associated to $\Pi \Theta$.

When the Lie group $S_{\omega}(2,2)$ is considered, two relevant families of symmetric homogeneous spaces [65] can be constructed as follows.

(i) The $(2+1) \mathrm{D}$ spacetime: this is a rank 1 space, associated with the automorphism $\Pi \Theta(4)$ and Cartan decomposition (5), which is defined through the quotient

$$
\mathbb{S}_{(1)}=\frac{S O_{\omega}(2,2)}{S O(2,1)},
$$

where $S O(2,1)$ is the Lorentz subgroup spanned by $J$ and $\mathbf{K}$. Thus momenta $P_{\mu}$ characterize the tangent space at the origin. This space turns out to have constant curvature equal to the contraction parameter: $\omega= \pm 1 / R^{2}$ for $(\mathrm{A}) \mathrm{dS}$ and $\omega=0(R \rightarrow$ $\infty)$ for Minkowski, where $R$ is the universe radius.

(ii) The $4 \mathrm{D}$ space of time-like lines (or wordlines): this is a rank 2 space, related to the automorphism $\Pi$ (3) and Cartan decomposition (6), which is given by

$$
\mathbb{S}_{(2)}=\frac{S O_{\omega}(2,2)}{\left(S O(2) \otimes S O_{\omega}(2)\right)},
$$

where $S O(2)=\langle J\rangle$ and $S O_{\omega}(2)=\left\langle P_{0}\right\rangle$. This space is of hyperbolic type as this has constant curvature equal to -1 (i.e., $-1 / c^{2}$ in terms of the speed of light). The tangent space is determined by spatial momenta $\mathbf{P}$ and boosts $\mathbf{K}$. In fact, $\mathbb{S}_{(2)}$ can also be interpreted as a $(2 \times 2) \mathrm{D}$ relativistic phase space [66] in which position and momentum coordinates are related to the group parameters dual to $\mathbf{P}$ and $\mathbf{K}$, respectively.

We display in Table 1 each of the above symmetrical homogeneous spaces for each of the three Lorentzian Lie groups.

On the other hand, the two Casimir invariants of the Lie algebra $s o_{\omega}(2,2)$ are given by

$$
\begin{aligned}
& \mathscr{C}=P_{0}^{2}-\mathbf{P}^{2}+\omega\left(J^{2}-\mathbf{K}^{2}\right), \\
& \mathscr{W}=-J P_{0}+K_{1} P_{2}-K_{2} P_{1},
\end{aligned}
$$

where $\mathbf{P}^{2}=P_{1}^{2}+P_{2}^{2}$ and $\mathbf{K}^{2}=K_{1}^{2}+K_{2}^{2}$. Recall that $\mathscr{C}$ comes from the Killing-Cartan form, while $\mathscr{W}$ is the Pauli-Lubanski vector.

2.1. Vector Model of the $(2+1) D$ Relativistic Spacetimes. The action of the (A)dS groups on the homogeneous spaces that 
we have just described is not linear. As it is well known, this problem can be circumvented by considering the vector representation of the Lie group $\operatorname{SO}_{\omega}(2,2)$ which makes use of an ambient space with an "extra" dimension. In particular, the $4 \mathrm{D}$ real matrix representation of $s o_{\omega}(2,2)$, given by

$$
\begin{aligned}
& P_{0}=\left(\begin{array}{cccc}
0 & -\omega & 0 & 0 \\
1 & 0 & 0 & 0 \\
0 & 0 & 0 & 0 \\
0 & 0 & 0 & 0
\end{array}\right), \\
& P_{1}=\left(\begin{array}{llll}
0 & 0 & \omega & 0 \\
0 & 0 & 0 & 0 \\
1 & 0 & 0 & 0 \\
0 & 0 & 0 & 0
\end{array}\right), \\
& P_{2}=\left(\begin{array}{llll}
0 & 0 & 0 & \omega \\
0 & 0 & 0 & 0 \\
0 & 0 & 0 & 0 \\
1 & 0 & 0 & 0
\end{array}\right), \\
& J=\left(\begin{array}{cccc}
0 & 0 & 0 & 0 \\
0 & 0 & 0 & 0 \\
0 & 0 & 0 & -1 \\
0 & 0 & 1 & 0
\end{array}\right), \\
& K_{1}=\left(\begin{array}{llll}
0 & 0 & 0 & 0 \\
0 & 0 & 1 & 0 \\
0 & 1 & 0 & 0 \\
0 & 0 & 0 & 0
\end{array}\right), \\
& K_{2}=\left(\begin{array}{llll}
0 & 0 & 0 & 0 \\
0 & 0 & 0 & 1 \\
0 & 0 & 0 & 0 \\
0 & 1 & 0 & 0
\end{array}\right),
\end{aligned}
$$

fulfils

$$
\begin{aligned}
Y^{T} \rrbracket_{(1)}+\rrbracket_{(1)} Y & =0, \\
Y & \in \operatorname{so}_{\omega}(2,2), \rrbracket_{(1)}=\operatorname{diag}(1, \omega,-\omega,-\omega)
\end{aligned}
$$

$\left(Y^{T}\right.$ is the transpose of $Y$ ). The exponential of (10) leads to the vector representation of $\mathrm{SO}_{\omega}(2,2)$ as a Lie group of matrices which acts linearly in a $4 \mathrm{D}$ space with ambient (or
Weierstrass) coordinates $\left(s_{3}, s_{\mu}\right)$. The one-parameter subgroups of $\mathrm{SO}_{\omega}(2,2)$ obtained from (10) turn out to be

$$
e^{x_{0} P_{0}}=\left(\begin{array}{cccc}
\cos \rho x_{0} & -\rho \sin \rho x_{0} & 0 & 0 \\
\frac{1}{\rho} \sin \rho x_{0} & \cos \rho x_{0} & 0 & 0 \\
0 & 0 & 1 & 0 \\
0 & 0 & 0 & 1
\end{array}\right)
$$$$
e^{\theta J}=\left(\begin{array}{cccc}
1 & 0 & 0 & 0 \\
0 & 1 & 0 & 0 \\
0 & 0 & \cos \theta & -\sin \theta \\
0 & 0 & \sin \theta & \cos \theta
\end{array}\right)
$$$$
e^{x_{1} P_{1}}=\left(\begin{array}{cccc}
\cosh \rho x_{1} & 0 & \rho \sinh \rho x_{1} & 0 \\
0 & 1 & 0 & 0 \\
\frac{1}{\rho} \sinh \rho x_{1} & 0 & \cosh \rho x_{1} & 0 \\
0 & 0 & 0 & 1
\end{array}\right) \text {, }
$$$$
e^{\xi_{1} K_{1}}=\left(\begin{array}{cccc}
1 & 0 & 0 & 0 \\
0 & \cosh \xi_{1} & \sinh \xi_{1} & 0 \\
0 & \sinh \xi_{1} & \cosh \xi_{1} & 0 \\
0 & 0 & 0 & 1
\end{array}\right),
$$$$
e^{x_{2} P_{2}}=\left(\begin{array}{cccc}
\cosh \rho x_{2} & 0 & 0 & \rho \sinh \rho x_{2} \\
0 & 1 & 0 & 0 \\
0 & 0 & 1 & 0 \\
\frac{1}{\rho} \sinh \rho x_{2} & 0 & 0 & \cosh \rho x_{2}
\end{array}\right) \text {, }
$$

$$
e^{\xi_{2} K_{2}}=\left(\begin{array}{cccc}
1 & 0 & 0 & 0 \\
0 & \cosh \xi_{2} & 0 & \sinh \xi_{2} \\
0 & 0 & 1 & 0 \\
0 & \sinh \xi_{2} & 0 & \cosh \xi_{2}
\end{array}\right)
$$

where hereafter we also express the curvature as $\omega=\rho^{2}$. Hence, $\rho=1 / R$ and $\rho=i / R$ for $\mathbf{A d} \mathbf{S}^{2+1}$ and $\mathbf{d} \mathbf{S}^{2+1}$, while the (contraction) limit $\rho \rightarrow 0$ gives rise to $\mathbf{M}^{2+1}$.

Any element $G \in S O_{\omega}(2,2)$ verifies that $G^{T} \rrbracket_{(1)} G=$ $\square_{(1)}$. The $(2+1) D$ spacetime $\mathbb{S}_{(1)}$ is identified with the orbit of the origin of the spacetime $O=\left(s_{3}, s_{\mu}\right)=(1,0,0,0)$ which is contained in the pseudosphere provided by $\rrbracket_{(1)}$ :

$$
\Sigma_{(1)}: s_{3}^{2}+\omega\left(s_{0}^{2}-s^{2}\right)=1,
$$


where $\mathbf{s}^{2}=s_{1}^{2}+s_{2}^{2}$. The metric on $\mathbb{S}_{(1)}$ comes from the flat ambient metric divided by the curvature and restricted to the above constraint:

$$
\begin{aligned}
\mathrm{d} \sigma_{(1)}^{2} & =\left.\frac{1}{\omega}\left(\mathrm{d} s_{3}^{2}+\omega\left(\mathrm{d} s_{0}^{2}-\mathrm{d} s_{1}^{2}-\mathrm{d} s_{2}^{2}\right)\right)\right|_{\Sigma_{(1)}} \\
& =\mathrm{d} s_{0}^{2}-\mathrm{d} s_{1}^{2}-\mathrm{d} s_{2}^{2}+\omega \frac{\left(s_{0} \mathrm{~d} s_{0}-s_{1} \mathrm{~d} s_{1}-s_{2} \mathrm{~d} s_{2}\right)^{2}}{1-\omega\left(s_{0}^{2}-\mathbf{s}^{2}\right)} .
\end{aligned}
$$

Ambient coordinates can be parametrized in terms of three intrinsic spacetime coordinates in different ways. We shall introduce the geodesic parallel coordinates $x_{\mu}$ [67] through the following action of the momenta subgroups (12) on the origin:

$$
\left(s_{3}, s_{\mu}\right)\left(x_{\nu}\right)=\exp \left(x_{0} P_{0}\right) \exp \left(x_{1} P_{1}\right) \exp \left(x_{2} P_{2}\right) O ;
$$

namely,

$$
\begin{aligned}
& s_{3}=\cos \rho x_{0} \cosh \rho x_{1} \cosh \rho x_{2}, \\
& s_{0}=\frac{\sin \rho x_{0}}{\rho} \cosh \rho x_{1} \cosh \rho x_{2}, \\
& s_{1}=\frac{\sinh \rho x_{1}}{\rho} \cosh \rho x_{2}, \\
& s_{2}=\frac{\sinh \rho x_{2}}{\rho} .
\end{aligned}
$$

The role of the coordinates $x_{\mu}$ that parametrize a generic point $Q$ under $(16)$ in the $(2+1) \mathrm{D}$ spacetime is the following. Let $l_{0}$ be a time-like geodesic and $l_{1}, l_{2}$ two space-like geodesics such that these three basic geodesics are orthogonal at $O$. Then $x_{0}$ is the geodesic distance from $O$ up to a point $Q_{1}$ measured along $l_{0} ; x_{1}$ is the distance between $Q_{1}$ and another point $Q_{2}$ along a space-like geodesic $l_{1}^{\prime}$ orthogonal to $l_{0}$ through $Q_{1}$ and parallel to $l_{1}$; and $x_{2}$ is the distance between $Q_{2}$ and $Q$ along a space-like geodesic $l_{2}^{\prime}$ orthogonal to $l_{1}^{\prime}$ and parallel to $l_{2}$. Recall that time-like geodesics (as $l_{0}$ ) are compact in $\mathbf{A d} \mathbf{S}^{2+1}$ and noncompact in $\mathbf{d} \mathbf{S}^{2+1}$, while spacelike ones (as $l_{i}, l_{i}^{\prime}$ ) are compact in $\mathbf{d} \mathbf{S}^{2+1}$ but noncompact in $\mathbf{A d S}^{2+1}$. Thus the trigonometric functions depending on $x_{0}$ are circular in $\mathbf{A d S}^{2+1}(\rho=1 / R)$ and hyperbolic in $\mathbf{d} \mathbf{S}^{2+1}$ $(\rho=i / R)$ and, conversely, those depending on $x_{i}$ are circular in $\mathbf{d} \mathbf{S}^{2+1}$ but hyperbolic in $\mathbf{A d} \mathbf{S}^{2+1}$.

Under (16), the metric (14) now reads

$$
\begin{aligned}
\mathrm{d} \sigma_{(1)}^{2}= & \cosh ^{2}\left(\rho x_{1}\right) \cosh ^{2}\left(\rho x_{2}\right) \mathrm{d} x_{0}^{2} \\
& -\cosh ^{2}\left(\rho x_{2}\right) \mathrm{d} x_{1}^{2}-\mathrm{d} x_{2}^{2} .
\end{aligned}
$$

Notice that if $\rho \rightarrow 0$, the parametrization (16) gives the flat Cartesian coordinates $s_{3}=1, s_{\mu}=x_{\mu}$, and the metric (17) reduces to $\mathrm{d} \sigma_{(1)}^{2}=\mathrm{d} x_{0}^{2}-\mathrm{d} x_{1}^{2}-\mathrm{d} x_{2}^{2}$ in $\mathbf{M}^{2+1}$.

2.2. Bivector Model of the 4D Spaces of Worldlines. The action of $S_{\omega}(2,2)$ on the space of time-like lines $\mathbb{S}_{(2)}$ is also a nonlinear one. As in the previous case, this problem can be solved by introducing an ambient space, now 6D with two "extra" dimensions, on which the group acts linearly and where $\mathbb{S}_{(2)}$ is embedded. Let us consider the so-called bivector representation of (2) given by [66]

$$
\begin{aligned}
P_{0} & =-\omega e_{24}+e_{42}-\omega e_{35}+e_{53}, \\
J & =-e_{23}+e_{32}-e_{45}+e_{54}, \\
P_{1} & =-\omega e_{14}-e_{41}+\omega e_{36}+e_{63}, \\
K_{1} & =e_{12}+e_{21}+e_{56}+e_{65}, \\
P_{2} & =-\omega e_{15}-e_{51}-\omega e_{26}-e_{62}, \\
K_{2} & =e_{13}+e_{31}-e_{46}-e_{64},
\end{aligned}
$$

where $e_{a b}$ is the $6 \times 6$ matrix with entries $\left(e_{a b}\right)_{i j}=\delta_{a i} \delta_{b j}$. Under this representation any generator $Y \in s o_{\omega}(2,2)$ fulfils

$$
\begin{aligned}
& Y^{T} \mathbb{\square}_{(2)}+\mathbb{\square}_{(2)} Y=0, \\
& \mathbb{\square}_{(2)}=\operatorname{diag}(1,-1,-1,-\omega,-\omega, \omega) .
\end{aligned}
$$

By exponentiation of (18) we obtain the bivector representation of $S_{\omega}(2,2)$, that is, a group of matrices which acts linearly in a $6 \mathrm{D}$ space with ambient (or Plücker) coordinates $\left(\eta_{3}, \eta_{1}, \eta_{2}, y_{1}, y_{2}, y_{3}\right)$. The origin of $\mathbb{S}_{(2)}$ is $\mathcal{O}=(1,0,0,0,0,0)$ and this space is identified with the intersection of the pseudosphere $\Sigma_{(2)}$ determined by $\rrbracket_{(2)}$ with a quadratic cone $\mathbb{P}$ known as Plücker or Grassmann relation (invariant under the group action); these constraints are given by [66]

$$
\begin{aligned}
& \Sigma_{(2)}: \eta_{3}^{2}-\boldsymbol{\eta}^{2}+\omega\left(y_{3}^{2}-\mathbf{y}^{2}\right)=1, \\
& \mathbb{P}: \eta_{3} y_{3}-\eta_{1} y_{2}+\eta_{2} y_{1}=0,
\end{aligned}
$$

where $\boldsymbol{\eta}^{2}=\eta_{1}^{2}+\eta_{2}^{2}$ and $\mathbf{y}^{2}=y_{1}^{2}+y_{2}^{2}$. The metric on $\mathbb{S}_{(2)}$ follows from the $6 \mathrm{D}$ flat ambient metric divided by the negative curvature of $\mathbb{S}_{(2)}$ and subjected to both conditions (20):

$$
\begin{aligned}
& \mathrm{d} \sigma_{(2)}^{2}=\frac{1}{-1}\left(\mathrm{~d} \eta_{3}^{2}-\mathrm{d} \eta_{1}^{2}-\mathrm{d} \eta_{2}^{2}\right. \\
& \left.\quad+\omega\left(\mathrm{d} y_{3}^{2}-\mathrm{d} y_{1}^{2}-\mathrm{d} y_{2}^{2}\right)\right)\left.\right|_{\Sigma_{(2)}, \mathbb{P}} .
\end{aligned}
$$

Plücker coordinates can be expressed through four intrinsic quantities of $\mathbb{S}_{(2)}$. We shall consider the space $\mathbf{x}$ and rapidity (boost) $\boldsymbol{\xi}$ group coordinates. The action of the following sequence of one-parameter subgroups on $\mathcal{O}$ (those defining the tangent space to $\mathbb{S}_{(2)}$ ) under the representation (18),

$$
\begin{aligned}
& \left(\eta_{3}, \boldsymbol{\eta}, \mathbf{y}, y_{3}\right)(\mathbf{x}, \boldsymbol{\xi}) \\
& \quad=\exp \left(x_{1} P_{1}\right) \exp \left(x_{2} P_{2}\right) \exp \left(\xi_{1} K_{1}\right) \exp \left(\xi_{2} K_{2}\right) \mathcal{O},
\end{aligned}
$$


gives rise to

$$
\begin{aligned}
\eta_{3}= & \cosh \rho x_{1} \cosh \rho x_{2} \cosh \xi_{1} \cosh \xi_{2}, \\
\eta_{1}= & \cosh \rho x_{2} \sinh \xi_{1} \cosh \xi_{2}, \\
\eta_{2}= & \cosh \rho x_{1} \sinh \xi_{2} \\
& -\sinh \rho x_{1} \sinh \rho x_{2} \sinh \xi_{1} \cosh \xi_{2}, \\
y_{1}= & -\frac{\sinh \rho x_{1}}{\rho} \cosh \rho x_{2} \cosh \xi_{1} \cosh \xi_{2}, \\
y_{2}= & -\frac{\sinh \rho x_{2}}{\rho} \cosh \xi_{1} \cosh \xi_{2}, \\
y_{3}= & \frac{\sinh \rho x_{1}}{\rho} \sinh \xi_{2} \\
& -\cosh \rho x_{1} \frac{\sinh \rho x_{2}}{\rho} \sinh \xi_{1} \cosh \xi_{2} .
\end{aligned}
$$

Under the contraction $\rho \rightarrow 0(\omega=0)$, this parametrization reduces to that of the $4 \mathrm{D}$ Minkowskian space of worldlines $\mathbf{L M}^{2 \times 2}$ :

$$
\begin{aligned}
& \eta_{3}=\cosh \xi_{1} \cosh \xi_{2}, \\
& y_{3}=x_{1} \sinh \xi_{2}-x_{2} \sinh \xi_{1} \cosh \xi_{2}, \\
& \eta_{1}=\sinh \xi_{1} \cosh \xi_{2}, \\
& y_{1}=-x_{1} \cosh \xi_{1} \cosh \xi_{2}, \\
& \eta_{2}=\sinh \xi_{2} \\
& y_{2}=-x_{2} \cosh \xi_{1} \cosh \xi_{2} .
\end{aligned}
$$

Such expressions indicate that the Plücker coordinates $\left(\mathbf{y}, y_{3}\right)$ and $\left(\boldsymbol{\eta}, \eta_{3}\right)$ can be interpreted as "position-like" and "momentum-like" ones, respectively, within the phase space $(\mathbf{x}, \boldsymbol{\xi})$ [66]. In $\mathbf{L M}^{2 \times 2}$ the metric (21) is degenerate and reads

$$
\begin{aligned}
\mathrm{d} \sigma_{(2)}^{2} & =\mathrm{d} \eta_{1}^{2}+\mathrm{d} \eta_{2}^{2}-\frac{\left(\eta_{1} \mathrm{~d} \eta_{1}+\eta_{2} \mathrm{~d} \eta_{2}\right)^{2}}{1+\boldsymbol{\eta}^{2}} \\
& =\cosh ^{2} \xi_{2} \mathrm{~d} \xi_{1}^{2}+\mathrm{d} \xi_{2}^{2},
\end{aligned}
$$

which corresponds to a $2 \mathrm{D}$ space of rank 1 with negative curvature; that is, the $2 \mathrm{D}$ velocity Minkowskian space (so with coordinates $\boldsymbol{\xi}$ ) is hyperbolic. Nevertheless, we stress that this is no longer true when $\omega \neq 0$ (in both LAdS $^{2 \times 2}$ and $\left.\mathbf{L} \mathbf{d} \mathbf{S}^{2 \times 2}\right)$ where the complete $(2 \times 2) \mathrm{D}$ space structure is required (so with the four coordinates $(\mathbf{x}, \boldsymbol{\xi})$ ), thus precluding the possibility of using a "reduced" $2 \mathrm{D}$ velocity space.

\section{3. (Anti-)de Sitter Drinfel'd-Doubles and First-Order Noncommutative Spaces}

The first-order deformation terms in the coproduct of the $\kappa$ Poincaré algebra $[9,13-18,22]$ are known to be generated by the following classical $r$-matrix:

$$
r=z\left(K_{1} \wedge P_{1}+K_{2} \wedge P_{2}\right)
$$

where $\wedge$ denotes the skew symmetric tensor product. Recall that $r$ is a solution of the modified classical Yang-Baxter equation and that $z$ is related to the usual $\kappa$ and $q$ deformation parameters by $z=1 / \kappa=\ln q$.

Such a classical $r$-matrix also holds for the (A)dS algebras [68], so that we shall consider (26) for the whole family so ${ }_{\omega}(2,2)$. Hence this element gives rise to the cocommutator $\delta$ of any generator $Y_{i}$ through the relation $\delta\left(Y_{i}\right)=\left[Y_{i} \otimes 1+1 \otimes\right.$ $\left.Y_{i}, r\right]$; namely,

$$
\begin{aligned}
\delta\left(P_{0}\right) & =0, \\
\delta(J) & =0, \\
\delta\left(P_{i}\right) & =z\left(P_{i} \wedge P_{0}-\omega \epsilon_{i j} K_{j} \wedge J\right), \\
\delta\left(K_{i}\right) & =z\left(K_{i} \wedge P_{0}+\epsilon_{i j} P_{j} \wedge J\right) .
\end{aligned}
$$

Next if we denote by $\widehat{y}^{i}$ the quantum group coordinate dual to $Y_{i}$, such that $\left\langle\hat{y}^{i} \mid Y_{j}\right\rangle=\delta_{j}^{i}$, and write the cocommutators as $\delta\left(Y_{i}\right)=f_{i}^{j k} Y_{j} \wedge Y_{k}$, then Lie bialgebra duality provides the so-called Drinfel'd-double Lie algebra $[7,8]$ formed by three sets of brackets: the initial Lie algebra, the dual relations, and the crossed commutation rules; namely,

$$
\begin{aligned}
& {\left[Y_{i}, Y_{j}\right]=c_{i j}^{k} Y_{k},} \\
& {\left[\hat{y}^{i}, \widehat{y}^{j}\right]=f_{k}^{i j} \widehat{y}^{k},} \\
& {\left[\widehat{y}^{i}, Y_{j}\right]=c_{j k}^{i} \hat{y}^{k}-f_{j}^{i k} Y_{k} .}
\end{aligned}
$$

The cocycle condition for the cocommutator $\delta$ implies the following compatibility equations among the structure constants $c_{i j}^{k}$ and $f_{k}^{i j}$ :

$$
f_{k}^{a b} c_{i j}^{k}=f_{i}^{a k} c_{k j}^{b}+f_{i}^{k b} c_{k j}^{a}+f_{j}^{a k} c_{i k}^{b}+f_{j}^{k b} c_{i k}^{a} .
$$

In our case, we denote by $\left\{\widehat{\theta}, \widehat{x}_{\mu}, \widehat{\xi}_{i}\right\}$ the dual noncommutative coordinates of the generators $\left\{J, P_{\mu}, K_{i}\right\}$, respectively. Thus the (A)dS and Poincaré Drinfel'd-doubles are collectively given in terms of the curvature $\omega$ and deformation parameter $z$ by the initial Lie algebra $s o_{\omega}(2,2)(2)$, the dual commutators,

$$
\begin{aligned}
{\left[\hat{\theta}, \widehat{x}_{i}\right] } & =z \epsilon_{i j} \widehat{\xi}_{j}, \\
{\left[\widehat{x}_{0}, \widehat{x}_{i}\right] } & =-z \widehat{x}_{i}, \\
{\left[\widehat{x}_{1}, \widehat{x}_{2}\right] } & =0, \\
{\left[\widehat{\theta}, \widehat{x}_{0}\right] } & =0, \\
{\left[\hat{\theta}, \widehat{\xi}_{i}\right] } & =-z \omega \epsilon_{i j} \widehat{x}_{j}, \\
{\left[\widehat{x}_{0}, \widehat{\xi}_{i}\right] } & =-z \widehat{\xi}_{i}, \\
{\left[\widehat{\xi}_{1}, \widehat{\xi}_{2}\right] } & =0, \\
{\left[\widehat{x}_{i}, \hat{\xi}_{j}\right] } & =0,
\end{aligned}
$$


together with the crossed relations

$$
\begin{aligned}
{\left[\widehat{x}_{0}, J\right] } & =\left[\widehat{x}_{0}, P_{0}\right]=0, \\
{[\widehat{\theta}, J] } & =\left[\widehat{\theta}, P_{0}\right]=0, \\
{\left[\widehat{x}_{0}, P_{i}\right] } & =-\left(\widehat{\xi}_{i}-z P_{i}\right), \\
{\left[\widehat{\theta}, P_{i}\right] } & =-\omega \epsilon_{i j}\left(\widehat{x}_{j}+z K_{j}\right), \\
{\left[\widehat{x}_{0}, K_{i}\right] } & =\widehat{x}_{i}+z K_{i}, \\
{\left[\widehat{\theta}_{,} K_{i}\right] } & =-\epsilon_{i j}\left(\widehat{\xi}_{j}-z P_{j}\right), \\
{\left[\widehat{x}_{i}, J\right] } & =-\epsilon_{i j} \widehat{x}_{j}, \\
{\left[\widehat{\xi}_{i}, J\right] } & =-\epsilon_{i j} \widehat{\xi}_{j}, \\
{\left[\widehat{x}_{i}, P_{0}\right] } & =-\widehat{\xi}_{i}, \\
{\left[\widehat{\xi}_{i}, P_{0}\right] } & =\omega \widehat{x}_{i}, \\
{\left[\widehat{x}_{i}, P_{j}\right] } & =\epsilon_{i j} \widehat{\theta}-z \delta_{i j} P_{0}, \\
{\left[\widehat{\xi}_{i}, P_{j}\right] } & =-\omega\left(\delta_{i j} \widehat{x}_{0}+z \epsilon_{i j} J\right), \\
{\left[\widehat{x}_{i}, K_{j}\right] } & =\delta_{i j} \widehat{x}_{0}+z \epsilon_{i j} J, \\
{\left[\hat{\xi}_{i}\right] } & =\epsilon_{i j} \widehat{\theta}_{-} z \delta_{i j} P_{0} .
\end{aligned}
$$

Parity and time-reversal automorphisms (3) can be generalized to the full Drinfel'd-double as follows:

$$
\begin{aligned}
\Pi_{z} & :\left(P_{0}, \mathbf{P}, \mathbf{K}, J ; \widehat{x}_{0}, \widehat{\mathbf{x}}, \widehat{\boldsymbol{\xi}}, \widehat{\theta} ; z\right) \\
& \longrightarrow\left(P_{0},-\mathbf{P},-\mathbf{K}, J ; \widehat{x}_{0},-\widehat{\mathbf{x}},-\widehat{\boldsymbol{\xi}}, \widehat{\theta} ; z\right), \\
\Theta_{z} & :\left(P_{0}, \mathbf{P}, \mathbf{K}, J ; \widehat{x}_{0}, \widehat{\mathbf{x}}, \widehat{\boldsymbol{\xi}}, \widehat{\theta} ; z\right) \\
& \longrightarrow\left(-P_{0}, \mathbf{P},-\mathbf{K}, J ;-\widehat{x}_{0}, \widehat{\mathbf{x}},-\widehat{\boldsymbol{\xi}}, \widehat{\theta} ;-z\right) .
\end{aligned}
$$

Since the first-order structure of the complete quantum deformation of $s o_{\omega}(2,2)$ is described by the corresponding Drinfel'd-double, some preliminary information concerning the physical properties of the associated noncommutative spaces can be extracted from it. Notice that, in this firstorder approach, all the expressions will be linear both on the generators and on the dual quantum group coordinates.

3.1. Noncommutative Spacetimes: Linear Relations. The usual way to propose a noncommutative spacetime is to consider the commutation rules involving the quantum coordinates $\widehat{x}_{\mu}$. Therefore, from (30) we find that the three (A)dS and Minkowskian noncommutative spacetimes are simultaneously defined by the same first-order relations:

$$
\begin{aligned}
& {\left[\widehat{x}_{0}, \widehat{x}_{i}\right]=-z \widehat{x}_{i},} \\
& {\left[\widehat{x}_{1}, \widehat{x}_{2}\right]=0,}
\end{aligned}
$$

which coincide with the $\kappa$-Minkowski space, $\mathbf{M}_{z}^{2+1}$ [15-18], for any value of the curvature $\omega$. As we shall see in Section 5, further corrections of (33) depending on $\omega$ will appear when the full quantum (A)dS groups are considered.

As it was already studied in $[29,30]$, the action of the generators on the noncommutative spacetime follows by replacing formally $P_{\mu} \rightarrow \widehat{x}_{\mu}$, which requires considering the commutators involving $\left\{J, \widehat{x}_{\mu}, K_{i}\right\}$ within the Drinfel'ddouble. Next the change of basis given by [30]

$$
\begin{aligned}
& \widehat{p}_{0}=-\widehat{x}_{0}, \\
& \widehat{p}_{i}=\widehat{x}_{i}+z K_{i},
\end{aligned}
$$

provides the following commutation relations:

$$
\begin{aligned}
{\left[J, \widehat{p}_{i}\right] } & =\epsilon_{i j} \widehat{p}_{j}, \\
{\left[J, K_{i}\right] } & =\epsilon_{i j} K_{j}, \\
{\left[J, \widehat{p}_{0}\right] } & =0, \\
{\left[\widehat{p}_{i}, K_{j}\right] } & =-\delta_{i j} \widehat{p}_{0}, \\
{\left[\widehat{p}_{0}, K_{i}\right] } & =-\widehat{p}_{i}, \\
{\left[K_{1}, K_{2}\right] } & =-J, \\
{\left[\widehat{p}_{0}, \widehat{p}_{i}\right] } & =-z^{2} K_{i}, \\
{\left[\widehat{p}_{1}, \widehat{p}_{2}\right] } & =z^{2} J
\end{aligned}
$$

that can directly be related to the initial Lie algebra (2). Consequently, whenever $z$ is a real deformation parameter, the commutators (35) that do not depend on $\omega$ close the Lie algebra $s o(3,1)$ for the three cases. Hence we obtain the dS spacetime as the homogeneous space

$$
\mathbf{d S}^{2+1} \equiv \frac{\left\langle J, \mathbf{K}, \widehat{p}_{\mu}\right\rangle}{\langle J, \mathbf{K}\rangle}=\frac{\operatorname{SO}(3,1)}{S O(2,1)},
$$

such that the deformation parameter now plays the role of the (negative) curvature equal to $-z^{2}$.

We stress that the connection between $\mathbf{M}_{z}^{2+1}$ and the $\mathrm{dS}$ space was so established in [29] and further developed in [30], so that the expressions (35) generalize such a link for the noncommutative $(\mathrm{A}) \mathrm{dS}$ cases as well.

3.2. Noncommutative Spaces of Worldlines: Linear Relations. A similar procedure suggests that the corresponding noncommutative spaces of worldlines arise within the Drinfel'ddouble through the commutators of $\widehat{\mathbf{x}}$ and $\widehat{\xi}$ (dual to $\mathbf{P}$ and $\mathbf{K})$; these are

$$
\begin{aligned}
& {\left[\widehat{x}_{1}, \widehat{x}_{2}\right]=0,} \\
& {\left[\widehat{\xi}_{1}, \widehat{\xi}_{2}\right]=0,} \\
& {\left[\widehat{x}_{i}, \widehat{\xi}_{j}\right]=0,}
\end{aligned}
$$

which are trivially independent of $z$ and $\omega$. 
The adjoint action on the quantum coordinates $\widehat{\mathbf{x}}, \widehat{\xi}$ of the isotropy subgroup of a worldline spanned by $J$ and $P_{0}(6)$ gives the following nondeformed commutation rules:

$$
\begin{aligned}
{\left[J, \widehat{x}_{i}\right] } & =\epsilon_{i j} \widehat{x}_{j}, \\
{\left[J, \widehat{\xi}_{i}\right] } & =\epsilon_{i j} \widehat{\xi}_{j}, \\
{\left[J, P_{0}\right] } & =0, \\
{\left[\widehat{x}_{i}, \widehat{\xi}_{j}\right] } & =0, \\
{\left[P_{0}, \widehat{\xi}_{i}\right] } & =-\omega \widehat{x}_{i}, \\
{\left[\widehat{\xi}_{1}, \widehat{\xi}_{2}\right] } & =0 \\
{\left[\widehat{x}_{1}, \widehat{x}_{2}\right] } & =0 \\
{\left[P_{0}, \widehat{x}_{i}\right] } & =\widehat{\xi}_{i},
\end{aligned}
$$

where the deformation parameter $z$ does not appear. To unveil this structure we rename the former generators as

$$
\begin{aligned}
J^{\prime} & =J, \\
P_{0}^{\prime} & =-P_{0}, \\
P_{i}^{\prime} & =\widehat{\xi}_{i}, \\
K_{i}^{\prime} & =\widehat{x}_{i},
\end{aligned}
$$

and from (38) we obtain the commutation relations

$$
\begin{aligned}
& {\left[J^{\prime}, P_{i}^{\prime}\right]=\epsilon_{i j} P_{j}^{\prime},} \\
& {\left[J^{\prime}, K_{i}^{\prime}\right]=\epsilon_{i j} K_{j}^{\prime},} \\
& {\left[J^{\prime}, P_{0}^{\prime}\right]=0,} \\
& {\left[P_{i}^{\prime}, K_{j}^{\prime}\right]=0,} \\
& {\left[P_{0}^{\prime}, K_{i}^{\prime}\right]=-P_{i}^{\prime},} \\
& {\left[K_{1}^{\prime}, K_{2}^{\prime}\right]=0,} \\
& {\left[P_{0}^{\prime}, P_{i}^{\prime}\right]=\omega K_{i}^{\prime},} \\
& {\left[P_{1}^{\prime}, P_{2}^{\prime}\right]=0 .}
\end{aligned}
$$

Surprisingly enough, these relations define just the Newtonian Lie algebras coming from the nonrelativistic limit $c \rightarrow$ $\infty$ of the three Lorentzian Lie algebras (2), keeping the constant curvature $\omega$. Namely, the relations (40) close the oscillating Newton-Hooke, Galilei, and expanding Newton-Hooke algebras $[19,53,63,69]$ according to $\omega>,=,<0$, respectively. This fact is consistent with the known result that establishes that each of the nonrelativistic Newtonian spaces of constant curvature $\omega$ can be obtained from the corresponding relativistic one through a contraction around a time-like line. Therefore, the classical (nondeformed) picture is preserved for worldlines.
Summing up, the first-order deformation of $s o_{\omega}(2,2)$ characterized by the chosen $r$-matrix (26) conveys noncommutativity on the spacetime (33) but commutativity on the space of worldlines (37). Furthermore, space isotropy is ensured in both cases as the corresponding commutation relations do not involve the quantum rotation coordinate $\widehat{\theta}$.

\section{A Poisson-Lie Structure on the (Anti-)de Sitter Groups}

So far we have studied the first-order quantum (A)dS deformation. However, the obtention of the complete (in all orders in $z$ and in the generators) deformation of a semisimple group in terms of local coordinates is, in general, a very involved task. A way to study the noncommutative structures is to compute the Poisson-Lie brackets (derived from (26)) for the commutative coordinates and next to analyse their possible noncommutative version.

In particular, let us consider the $4 \times 4$ matrix element of the group $\mathrm{SO}_{\omega}(2,2)$ obtained through the following product written under the representation (12):

$$
\begin{aligned}
T= & \exp \left(x_{0} P_{0}\right) \exp \left(x_{1} P_{1}\right) \exp \left(x_{2} P_{2}\right) \exp \left(\xi_{1} K_{1}\right) \\
& \cdot \exp \left(\xi_{2} K_{2}\right) \exp (\theta J)
\end{aligned}
$$

where the group coordinates are commutative ones. Leftand right-invariant vector fields, $Y^{L}$ and $Y^{R}$, of $S_{\omega}(2,2)$ deduced from (41) are displayed in Table 2. Notice that such expressions hold for any value of the curvature $\omega$. In the Poincaré case with $\omega=0$, the vector fields, coming from the smooth contraction limit $\rho \rightarrow 0$, are rather simplified as shown in Table 3.

The Poisson-Lie brackets that close the algebra of smooth functions on the (A)dS groups, $\operatorname{Fun}\left(\mathrm{SO}_{\omega}(2,2)\right)$ (r.h.s. of the diagram (1)), associated to an $r$-matrix $r=r^{i j} Y_{i} \otimes Y_{j}$ come from the Sklyanin bracket defined by [70]

$$
\begin{aligned}
\{f, g\}=r^{i j}\left(Y_{i}^{L} f Y_{j}^{L} g-Y_{i}^{R} f Y_{j}^{R} g\right) & \\
\qquad & f, g \in \operatorname{Fun}\left(S O_{\omega}(2,2)\right) .
\end{aligned}
$$

Thus by substituting the vector fields of Table 2 and the classical $r$-matrix (26) in (42) we obtain the Poisson-Lie brackets between the six commutative group coordinates $\left\{\theta, x_{\mu}, \xi_{i}\right\}$ which are split in the following three sets:

(i) those involving spacetime $x_{\mu}$ group coordinates,

$$
\begin{aligned}
& \left\{x_{0}, x_{1}\right\}=-z \frac{\tanh \rho x_{1}}{\rho \cosh ^{2} \rho x_{2}} \\
& \left\{x_{0}, x_{2}\right\}=-z \frac{\tanh \rho x_{2}}{\rho} \\
& \left\{x_{1}, x_{2}\right\}=0
\end{aligned}
$$




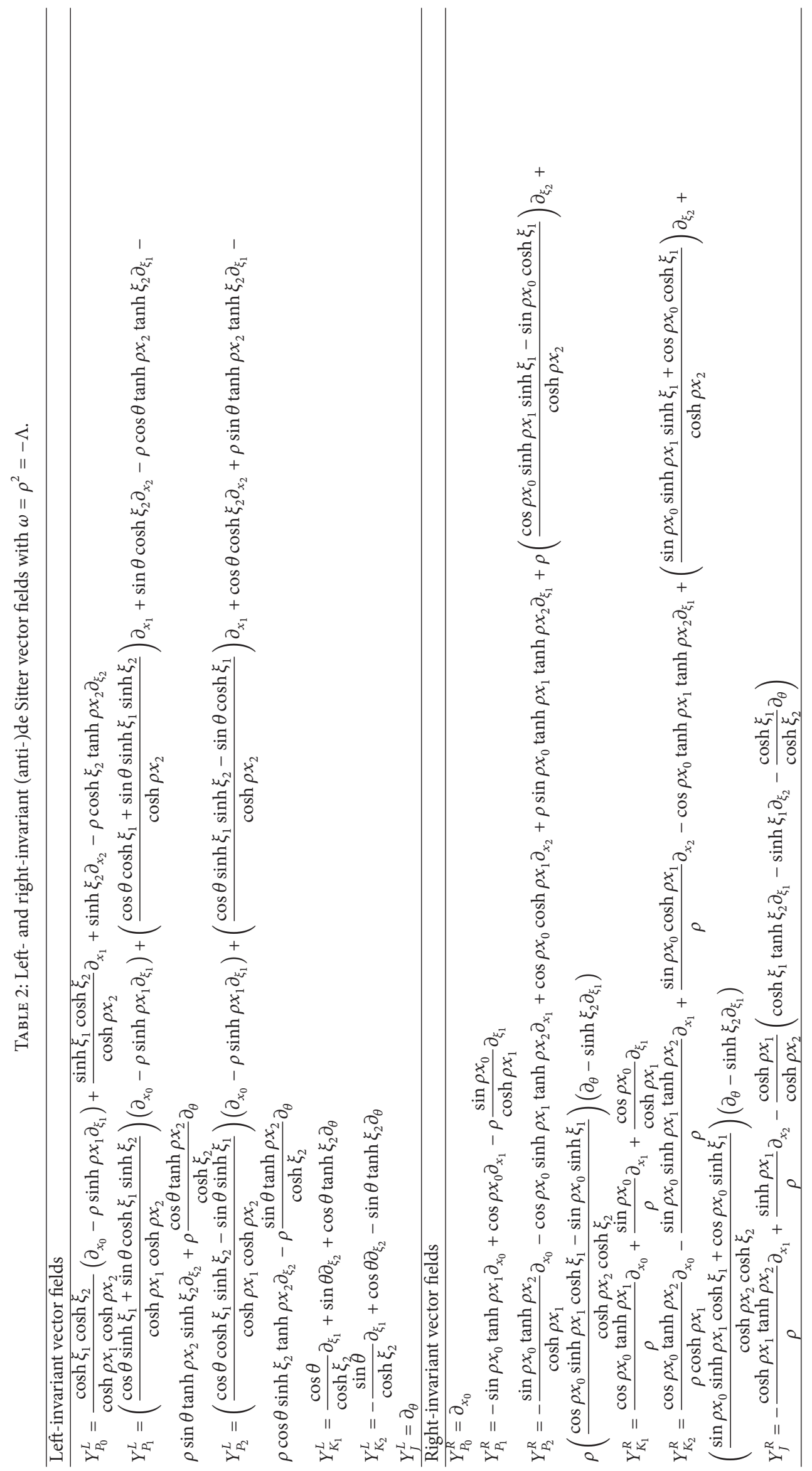


TABLE 3: Left- and right-invariant Poincaré vector fields with $\omega=\rho=\Lambda=0$.

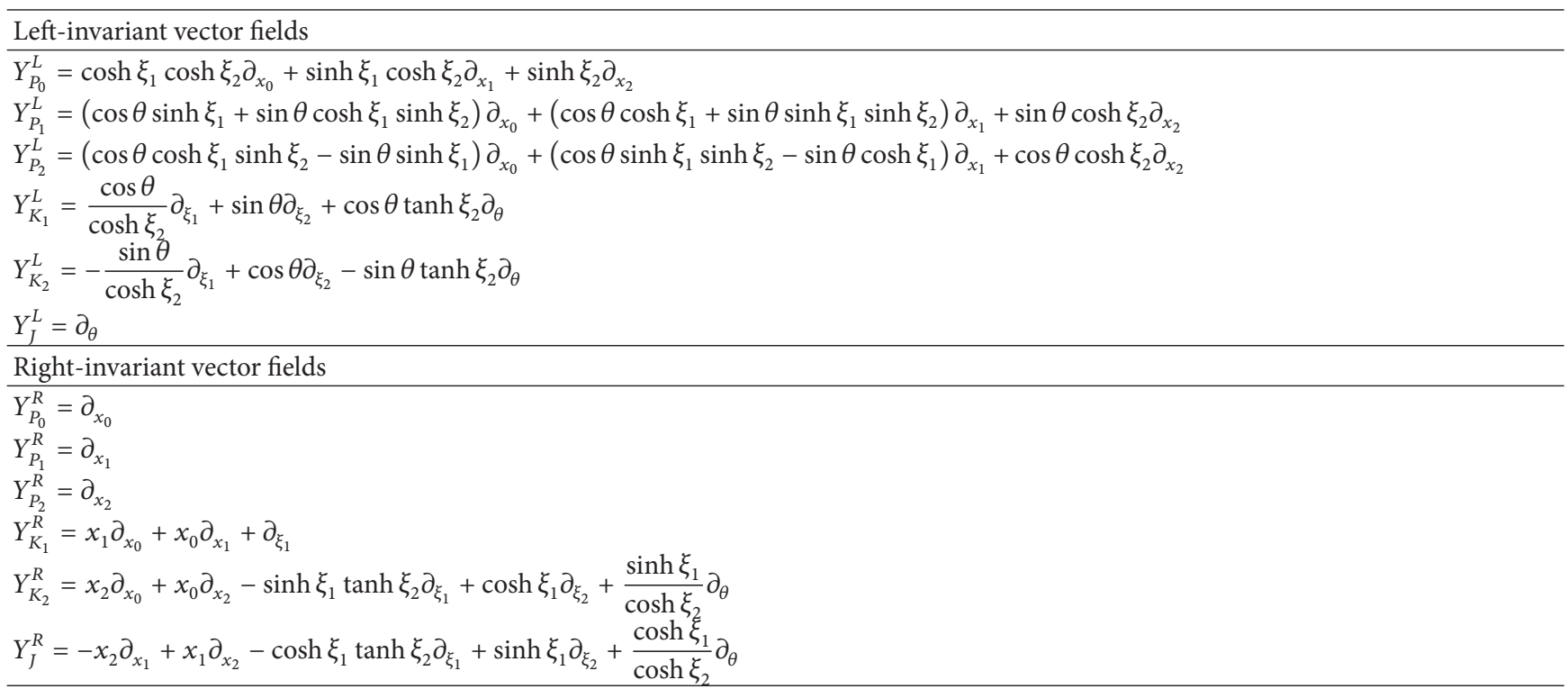

(ii) those that comprise space $\mathbf{x}$ and boost $\boldsymbol{\xi}$ coordinates (besides the above vanishing bracket),

$$
\begin{aligned}
& \left\{x_{1}, \xi_{1}\right\}=\frac{z}{\cosh \rho x_{2}}\left(\frac{\cosh \rho x_{2}}{\cosh \rho x_{1}}-\frac{\cosh \xi_{1}}{\cosh \xi_{2}}\right. \\
& \left.+\tanh \rho x_{1} \sinh \rho x_{2} A\right), \\
& \left\{x_{1}, \xi_{2}\right\}=-z \cosh \xi_{2} B, \\
& \left\{x_{2}, \xi_{2}\right\}=z\left(\frac{\cosh \rho x_{1}}{\cosh \rho x_{2}} \cosh \xi_{1}-\cosh \xi_{2}\right), \\
& \left\{x_{2}, \xi_{1}\right\}=-z A, \\
& \left\{\xi_{1}, \xi_{2}\right\}=z \rho \sinh \rho x_{1}\left(C-\frac{\tanh _{2}}{\cosh ^{2} \rho x_{2}}\right),
\end{aligned}
$$

(iii) the remaining ones,

$$
\begin{aligned}
\left\{x_{0}, \theta\right\} & =-\frac{z}{\cosh \rho x_{1}} B, \\
\left\{x_{0}, \xi_{1}\right\} & =z\left(\frac{\sinh \xi_{2}}{\cosh \rho x_{1}} B-\frac{\sinh \xi_{1} \cosh \xi_{2}}{\cosh \rho x_{1} \cosh \rho x_{2}}\right) \\
\left\{x_{0}, \xi_{2}\right\} & =-z C \\
\left\{\theta, x_{1}\right\} & =z \frac{\cosh \rho x_{1}}{\cosh \xi_{2}} C \\
\left\{\theta, x_{2}\right\} & =-z \frac{\cosh \rho x_{1} \sinh \xi_{1}}{\cosh \rho x_{2} \cosh \xi_{2}} \\
\left\{\theta, \xi_{1}\right\} & =-z \rho\left(\tanh \rho x_{2}+\tanh \rho x_{1} B\right) \\
\left\{\theta, \xi_{2}\right\} & =\frac{z \rho \sinh \rho x_{1}}{\cosh \rho x_{2} \cosh \xi_{2}}
\end{aligned}
$$

where the functions $A, B$, and $C$ are

$$
\begin{aligned}
& A=\frac{\sinh \rho x_{1} \sinh \rho x_{2}+\cosh \rho x_{1} \sinh \xi_{1} \tanh \xi_{2}}{\cosh \rho x_{2}}, \\
& B=\frac{\sinh \rho x_{1} \tanh \rho x_{2} \cosh \xi_{1}+\sinh \xi_{1} \sinh \xi_{2}}{\cosh \rho x_{2} \cosh \xi_{2}} \\
& C=\frac{\sinh \rho x_{1} \tanh \rho x_{2} \sinh \xi_{1}+\cosh \xi_{1} \sinh \xi_{2}}{\cosh \rho x_{1} \cosh \rho x_{2}}
\end{aligned}
$$

Notice that the commutators (30) are recovered from (43)-(46) by taking the first-order in the group coordinates.

We remark that any other choice for the matrix element of $S O_{\omega}(2,2)$ would lead to another set of vector fields formally different from those written in Table 2 and, therefore, it would give rise to Poisson-Lie brackets different from (43)-(46). However, by construction, all of these possible vector fields and Poisson-Lie structures are equivalent by means of changes of basis involving the group coordinates.

\section{Noncommutative (Anti-)de Sitter Spaces}

In general, the simplest way to quantize Poisson-Lie structures $[5,70]$ consists in the usual Weyl substitution of the initial Poisson brackets between commutative coordinates by commutators between noncommutative coordinates. Several quantum deformations of nonsemisimple groups have been constructed by applying this procedure; among them we underline the $\kappa$-Poincaré group [15-18], for which the full set of commutation rules are linear in the deformation parameter. Nevertheless, we stress that a quantum group does not always coincide with the Weyl quantization of their underlying Poisson-Lie brackets, specially when dealing with semisimple groups as the (A)dS ones, since ordering problems often appear during the quantization procedure. 
In our case, the Poisson-Lie brackets (43)-(46) are the commutative counterpart of the full noncommutative quantum (A)dS groups $\operatorname{Fun}_{z}\left(\mathrm{SO}_{\omega}(2,2)\right)$. Thus if we write a generic Poisson-Lie bracket as

$$
\left\{y^{i}, y^{j}\right\}=z f\left(y^{1}, \ldots, y^{6}\right)
$$

its noncommutative version would read

$$
\left[\hat{y}^{i}, \hat{y}^{j}\right]=z f\left(\hat{y}^{1}, \ldots, \hat{y}^{6}\right)+o\left(z^{2}\right)
$$

whose first-order in all $\hat{y}^{k}$ and $z$ is given by (30), while the $o\left(z^{2}\right)$ terms come from the reordering of the quantum coordinates $\widehat{y}^{k}$. By following this point of view, we will analyse the generalization of the first-order noncommutative spaces presented in Section 3 to higher orders in the quantum coordinates and up to second-order in $z$.

5.1. Noncommutative Spacetimes. The first set of Poisson-Lie brackets (43) allows us to introduce the defining commutation relations of the $(2+1) \mathrm{D}$ noncommutative (A)dS spacetimes; namely,

$$
\begin{aligned}
{\left[\widehat{x}_{0}, \widehat{x}_{1}\right] } & =-z \frac{\tanh \rho \widehat{x}_{1}}{\rho \cosh ^{2} \rho \widehat{x}_{2}}+o\left(z^{2}\right) \\
& =-z \widehat{x}_{1}+\frac{1}{3} z \omega \widehat{x}_{1}^{3}+z \omega \widehat{x}_{1} \widehat{x}_{2}^{2}+o\left(z^{2}\right), \\
{\left[\widehat{x}_{0}, \widehat{x}_{2}\right] } & =-z \frac{\tanh \rho \widehat{x}_{2}}{\rho}+o\left(z^{2}\right) \\
& =-z \widehat{x}_{2}+\frac{1}{3} z \omega \widehat{x}_{2}^{3}+o\left(z^{2}\right), \\
{\left[\widehat{x}_{1}, \widehat{x}_{2}\right] } & =0+o\left(z^{2}\right) .
\end{aligned}
$$

These expressions demonstrate how the underlying firstorder $\mathbf{M}_{z}^{2+1}$ (33) for the (A)dS groups is now generalized with an explicit dependence on the curvature $\omega$, in such a way that three different cases appear. Furthermore, space isotropy is preserved in the quantum case since $\hat{\theta}$ is again absent (as well as the quantum boost coordinates $\widehat{\xi}$ ).

It is worth mentioning that the asymmetric form of (49) could be expected from the beginning (see, e.g., the classical metric (17)) as we are dealing with local quantum coordinates. However, if we consider noncommutative ambient (Weierstrass) coordinates $\left(\widehat{s}_{3}, \widehat{s}_{\mu}\right)$ defined in terms of the former ones $\hat{x}_{\mu}$ by the same formal relations (16) and subjected to the constraint (13), we obtain the noncommutative spacetimes written in a fully symmetric way as a quadratic algebra:

$$
\begin{aligned}
& {\left[\widehat{s}_{0}, \widehat{s}_{i}\right]=-z \widehat{s}_{3} \widehat{s}_{i}+o\left(z^{2}\right),} \\
& {\left[\widehat{s}_{1}, \widehat{s}_{2}\right]=0+o\left(z^{2}\right),} \\
& {\left[\widehat{s}_{3}, \widehat{s}_{0}\right]=z w \widehat{\mathbf{s}}^{2}+o\left(z^{2}\right),} \\
& {\left[\widehat{s}_{3}, \widehat{s}_{i}\right]=z w \widehat{s}_{0} \widehat{s}_{i}+o\left(z^{2}\right) .}
\end{aligned}
$$

These expressions are clearly invariant under the permutation $\widehat{s}_{1} \leftrightarrow \widehat{s}_{2}$. The two first relations in (50) are just $\mathbf{M}_{z}^{2+1}$, since $\widehat{s}_{3} \rightarrow 1$ when $\omega \rightarrow 0$.

5.2. Noncommutative Spaces of Worldlines. Likewise, a first insight into the noncommutative (A)dS and Minkowskian spaces of time-like lines can be performed by starting with the Poisson-Lie brackets (44) among space $\mathbf{x}$ and boost $\boldsymbol{\xi}$ group coordinates.

Let us consider firstly the quantum Poincaré group with $\omega=0$. In this case, the expressions (44) are rather simplified. Their corresponding quantum deformation reads

$$
\begin{aligned}
& {\left[\widehat{x}_{1}, \hat{\xi}_{1}\right]=z\left(1-\frac{\cosh \widehat{\xi}_{1}}{\cosh \widehat{\xi}_{2}}\right),} \\
& {\left[\widehat{x}_{2}, \widehat{\xi}_{2}\right]=z\left(\cosh \widehat{\xi}_{1}-\cosh \widehat{\xi}_{2}\right),} \\
& {\left[\widehat{x}_{1}, \widehat{\xi}_{2}\right]=-z \sinh \widehat{\xi}_{1} \sinh \widehat{\xi}_{2},} \\
& {\left[\widehat{x}_{2}, \widehat{\xi}_{1}\right]=-z \sinh \widehat{\xi}_{1} \tanh \widehat{\xi}_{2},} \\
& {\left[\widehat{x}_{1}, \widehat{x}_{2}\right]=0,} \\
& {\left[\widehat{\xi}_{1}, \widehat{\xi}_{2}\right]=0,}
\end{aligned}
$$

which determine the complete (in all orders in $z, \widehat{\mathbf{x}}$, and $\widehat{\boldsymbol{\xi}}$ ) noncommutative Minkowskian space of worldlines $\mathbf{L M}_{z}^{2 \times 2}$. Hence the commutativity of the first-order relations $\left[\widehat{x}_{i}, \widehat{\xi}_{j}\right]$ is lost (see (37)). It is worth mentioning that $\left[\widehat{\xi}_{1}, \widehat{\xi}_{2}\right]=0$ ensures the self-consistency of the remaining commutators as no ordering problems appear. Moreover, this condition means that the $2 \mathrm{D}$ velocity space remains nondeformed (commutative).

On the contrary, in the (A)dS cases with $\omega \neq 0$ the brackets (44) are rather involved, again asymmetric, and $\widehat{\xi}_{1}, \widehat{\xi}_{2}$ no longer commute. As in the classical case, we can consider the noncommutative ambient (Plücker) coordinates $\left(\widehat{\eta}_{3}, \widehat{\boldsymbol{\eta}}, \widehat{\mathbf{y}}, \widehat{y}_{3}\right)$ formally defined by (23), but now with noncommutative entries $\widehat{\mathbf{x}}$ and $\widehat{\boldsymbol{\xi}}$. Then the noncommutative (A)dS spaces of worldlines are given by the following quadratic relations:

$$
\begin{aligned}
{\left[\widehat{y}_{1}, \widehat{y}_{2}\right]=} & -z\left(\widehat{\eta}_{3}-\left(\widehat{\eta}_{3}^{2}-\omega \widehat{\mathbf{y}}^{2}\right)\right) \widehat{y}_{3}+o\left(z^{2}\right), \\
{\left[\widehat{y}_{3}, \widehat{\eta}_{3}\right]=} & 0+o\left(z^{2}\right), \\
{\left[\widehat{\eta}_{1}, \widehat{\eta}_{2}\right]=} & -z \omega\left(\widehat{\eta}_{3}-\left(\widehat{\eta}_{3}^{2}-\omega \widehat{\mathbf{y}}^{2}\right)\right)\left(\frac{\widehat{\eta}_{3}-1}{\widehat{\eta}_{3}}\right) \widehat{y}_{3} \\
& +o\left(z^{2}\right), \\
{\left[\widehat{\eta}_{3}, \widehat{\eta}_{i}\right]=} & z \omega\left(\epsilon_{i j} \widehat{\eta}_{j} \widehat{y}_{3}+\widehat{y}_{i}\left(\widehat{\eta}_{3}^{2}-\omega \widehat{\mathbf{y}}^{2}\right)\left(\frac{\widehat{\eta}_{3}-1}{\widehat{\eta}_{3}}\right)\right) \\
& +o\left(z^{2}\right),
\end{aligned}
$$




$$
\begin{aligned}
& {\left[\hat{\eta}_{3}, \hat{y}_{i}\right]=z\left(\omega \epsilon_{i j} \hat{y}_{j} \hat{y}_{3}-\hat{\eta}_{i}\left(\hat{\eta}_{3}^{2}-\omega \hat{\mathbf{y}}^{2}\right)\left(\frac{\hat{\eta}_{3}-1}{\hat{\eta}_{3}}\right)\right)} \\
& +o\left(z^{2}\right) \\
& {\left[\hat{y}_{3}, \hat{y}_{i}\right]=z\left(\epsilon_{i j} \widehat{y}_{j}\left(\widehat{\eta}_{3}-1\right)-\widehat{\eta}_{i}\left(\widehat{\eta}_{3}^{2}-\omega \widehat{\mathbf{y}}^{2}\right) \frac{\widehat{y}_{3}}{\widehat{\eta}_{3}}\right)} \\
& +o\left(z^{2}\right) \\
& {\left[\hat{y}_{3}, \hat{\eta}_{i}\right]=z\left(\epsilon_{i j} \hat{\eta}_{j}\left(\hat{\eta}_{3}-1\right)+\omega \hat{y}_{i}\left(\widehat{\eta}_{3}^{2}-\omega \widehat{\mathbf{y}}^{2}\right) \frac{\hat{y}_{3}}{\widehat{\eta}_{3}}\right)} \\
& +o\left(z^{2}\right) \\
& {\left[\widehat{\eta}_{i}, \widehat{y}_{j}\right]=z \delta_{i j} \widehat{\eta}_{3}\left(\widehat{\eta}_{3}-1+\omega \hat{y}_{3}^{2}\right)-z \widehat{\eta}_{i} \hat{\eta}_{j} \widehat{\eta}_{3}} \\
& +z \omega \widehat{y}_{i} \widehat{y}_{j}\left(\omega \frac{\hat{y}_{3}^{2}}{\hat{\eta}_{3}}-1\right) \\
& +z \omega\left(\epsilon_{i k} \widehat{\eta}_{k} \hat{y}_{j}\left(\frac{\widehat{\eta}_{3}-1}{\hat{\eta}_{3}}\right)+\epsilon_{j k} \widehat{\eta}_{k} \hat{y}_{i}\right) \hat{y}_{3} \\
& +o\left(z^{2}\right)
\end{aligned}
$$

which are invariant under the map defined by

$$
\left(\widehat{\eta}_{3}, \hat{\eta}_{1}, \hat{\eta}_{2}, \hat{y}_{1}, \hat{y}_{2}, \hat{y}_{3}\right) \longrightarrow\left(\hat{\eta}_{3}, \hat{\eta}_{2}, \widehat{\eta}_{1}, \hat{y}_{2}, \hat{y}_{1},-\widehat{y}_{3}\right) .
$$

The flat contraction $\omega=0$ of (52) to $\mathbf{L M}_{z}^{2 \times 2}$ yields

$$
\begin{aligned}
& {\left[\widehat{y}_{1}, \hat{y}_{2}\right]=z \hat{y}_{3} \widehat{\eta}_{3}\left(\widehat{\eta}_{3}-1\right) \text {, }} \\
& {\left[\hat{y}_{3}, \widehat{\eta}_{3}\right]=0 \text {, }} \\
& {\left[\widehat{\eta}_{1}, \widehat{\eta}_{2}\right]=0 \text {, }} \\
& {\left[\hat{\eta}_{3}, \widehat{\eta}_{i}\right]=0 \text {, }} \\
& {\left[\hat{\eta}_{3}, \hat{y}_{i}\right]=-z \widehat{\eta}_{i} \hat{\eta}_{3}\left(\widehat{\eta}_{3}-1\right),} \\
& {\left[\hat{y}_{3}, \hat{y}_{i}\right]=z \epsilon_{i j} \hat{y}_{j}\left(\hat{\eta}_{3}-1\right)-z \hat{\eta}_{i} \hat{\eta}_{3} \hat{y}_{3}+o\left(z^{2}\right) \text {, }} \\
& {\left[\hat{y}_{3}, \widehat{\eta}_{i}\right]=z \epsilon_{i j} \widehat{\eta}_{j}\left(\widehat{\eta}_{3}-1\right) \text {, }} \\
& {\left[\widehat{\eta}_{i}, \widehat{y}_{j}\right]=z \delta_{i j} \widehat{\eta}_{3}\left(\widehat{\eta}_{3}-1\right)-z \widehat{\eta}_{i} \widehat{\eta}_{j} \widehat{\eta}_{3} .}
\end{aligned}
$$

The commutativity of the $2 \mathrm{D}$ velocity space in $\mathbf{L M}_{z}^{2 \times 2}$ now comes from the fact that the commutators involving the "momentum-like" coordinates $\left(\widehat{\eta}_{3}, \widehat{\eta}\right)$ vanish; note that they only depend on $\widehat{\xi}$, which commute in this case. On the contrary, the "position-like" ones $\left(\hat{y}_{3}, \widehat{\mathbf{y}}\right)$ do not commute; they depend on both $\widehat{\xi}$ and $\widehat{\mathbf{x}}$ (see (24)). We stress that this quantum Poincaré group property is in full agreement with the study developed in $[71,72]$ by working with the (dual) quantum algebra. In these works it is shown how the $\kappa$ deformed Poincaré boost transformations close a group as in the nondeformed case, and the additivity of the boost parameter for transformations along the same direction is also preserved. Therefore, the relations (52) indicate that such properties may be either lost or somewhat modified in the quantum $(\mathrm{A}) \mathrm{dS}$ groups.

\section{Quantum (Anti-)de Sitter Algebras}

In this section we firstly review the Hopf algebra structure and the associated invariants of the quantum (A)dS algebras that quantize the cocommutators (27), commutators (2), and Casimirs (9). These results are presented in the kinematical basis in which they were formerly obtained [19], and the contraction $\omega=0$ gives the $\kappa$-Poincaré written in the form deduced in [13]. We also point out some remarks concerning the connection between these structures and quantum gravity that has been introduced in [38]. Secondly, we obtain a new basis through a nonlinear map for these quantum algebras in such a manner that the $\kappa$-Poincare algebra is recovered in the so-called bicrossproduct basis $[8,16]$. This change of basis, at the level of the quantum algebra, is the dual counterpart of a change of noncommutative coordinates on the quantum group.

6.1. "Symmetrical" Basis. The Drinfel'd-Jimbo quantum deformation of $s o(4, \mathbb{C})$ was obtained in [11] by considering two copies of the quantum $\operatorname{sl}(2)$ algebra [3] and applying the prescription $U_{z}(s o(4, \mathbb{C}))=U_{z}(s l(2)) \oplus U_{-z}(s l(2))$. From this result, a further analysis of real forms together with a contraction scheme led to quantum deformations of the family of $(2+1) D$ kinematical algebras $[19,68]$ which included, among others, the $\kappa$-deformation of the three relativistic algebras (2) with underlying Lie bialgebra (27) that we denote here by $U_{z}\left(s o_{\omega}(2,2)\right)$. The Hopf structure of $U_{z}\left(s o_{\omega}(2,2)\right)$ is characterized by the following coproduct and commutation relations [19]:

$$
\begin{aligned}
\Delta\left(P_{0}\right)= & 1 \otimes P_{0}+P_{0} \otimes 1 \\
\Delta(J)= & 1 \otimes J+J \otimes 1, \\
\Delta\left(P_{i}\right)= & e^{-(z / 2) P_{0}} \cosh \left(\frac{z}{2} \rho J\right) \otimes P_{i}+P_{i} \\
& \otimes e^{(z / 2) P_{0}} \cosh \left(\frac{z}{2} \rho J\right) \\
& +\rho e^{-(z / 2) P_{0}} \sinh \left(\frac{z}{2} \rho J\right) \otimes \epsilon_{i j} K_{j} \\
& -\rho \epsilon_{i j} K_{j} \otimes e^{(z / 2) P_{0}} \sinh \left(\frac{z}{2} \rho J\right), \\
\Delta\left(K_{i}\right)= & e^{-(z / 2) P_{0}} \cosh \left(\frac{z}{2} \rho J\right) \otimes K_{i}+K_{i} \\
& \otimes e^{(z / 2) P_{0}} \cosh \left(\frac{z}{2} \rho J\right) \\
& -e^{-(z / 2) P_{0}} \frac{\sinh ((z / 2) \rho J)}{\rho} \otimes \epsilon_{i j} P_{j} \\
& +\epsilon_{i j} P_{j} \otimes e^{(z / 2) P_{0}} \frac{\sinh ((z / 2) \rho J)}{\rho},
\end{aligned}
$$




$$
\begin{aligned}
{\left[J, P_{i}\right] } & =\epsilon_{i j} P_{j}, \\
{\left[J, K_{i}\right] } & =\epsilon_{i j} K_{j}, \\
{\left[J, P_{0}\right] } & =0 \\
{\left[P_{i}, K_{j}\right] } & =-\delta_{i j} \frac{\sinh \left(z P_{0}\right)}{z} \cosh (z \rho J), \\
{\left[P_{0}, K_{i}\right] } & =-P_{i}, \\
{\left[P_{0}, P_{i}\right] } & =\omega K_{i}, \\
{\left[P_{1}, P_{2}\right] } & =-\omega \cosh \left(z P_{0}\right) \frac{\sinh (z \rho J)}{z \rho}, \\
{\left[K_{1}, K_{2}\right] } & =-\cosh \left(z P_{0}\right) \frac{\sinh (z \rho J)}{z \rho} .
\end{aligned}
$$

Counit and antipode maps can directly be derived from the Hopf algebra axioms. The deformation of the two Casimir invariants (9) turns out to be

$$
\begin{aligned}
\mathscr{C} & =4 \cos (z \rho)\left\{\frac{\sinh ^{2}\left((z / 2) P_{0}\right)}{z^{2}} \cosh ^{2}\left(\frac{z}{2} \rho J\right)\right. \\
& \left.+\frac{\sinh ^{2}((z / 2) \rho J)}{z^{2}} \cosh ^{2}\left(\frac{z}{2} P_{0}\right)\right\}-\frac{\sin (z \rho)}{z \rho}\left(\mathbf{P}^{2}\right. \\
& \left.+\omega \mathbf{K}^{2}\right), \\
\mathscr{W} & =-\cos (z \rho) \frac{\sinh (z \rho J)}{z \rho} \frac{\sinh \left(z P_{0}\right)}{z} \\
& +\frac{\sin (z \rho)}{z \rho}\left(K_{1} P_{2}-K_{2} P_{1}\right) .
\end{aligned}
$$

This deformation is governed by the generators spanning the isotropy subgroup of a worldline, $P_{0}$ and $J$, which remain nondeformed at the level of the coproduct (55). Thus deformed functions of $P_{0}$ and $J$ arise for the coproduct of $\mathbf{P}$ and $\mathbf{K}$ in both spaces in the tensor product, in such a manner that their coproduct is invariant under the composition $\sigma \circ \mathscr{T}$ of the flip operator, $\sigma(x \otimes y)=y \otimes x$, and a "parity" operator $\mathscr{T}$ acting on the deformation parameter as $\mathscr{T}(z)=-z$. Hence we say that $U_{z}\left(s o_{\omega}(2,2)\right)$ is written in a "symmetrical" basis.

The physical dimension of $z$ is inherited from $P_{0},[z]=$ $\left[P_{0}\right]^{-1}$, so that this can be interpreted as a fundamental length (provided that $c=1$ ), which in the usual DSR theories is considered to be of the order of the Planck length $l_{p}$.

Expressions (55)-(57) show the commutativity character of the 1.h.s. of the diagram (1); the limit $z \rightarrow 0$ in each of the three particular quantum algebras contained in the family $U_{z}\left(s o_{\omega}(2,2)\right)$ leads to the corresponding Lie algebra $s o_{\omega}(2,2)$ and its invariants, while the contraction $\omega=0$ $(\rho \rightarrow 0)$ in $U_{z}\left(s o_{\omega}(2,2)\right)$ gives rise to the $\kappa$-Poincaré algebra and its deformed invariants in the basis formerly worked out in [13]. Both types of limits can be applied separately. Such a viewpoint suggests some kind of "duality" between quantum deformations induced by $z$ and "curvature deformations" parametrized by $\omega$; recall that (A)dS symmetries can be considered as a "classical" deformation of Poincaré invariance $[52,53,65]$.

The dual relationship between quantum deformation parameters and classical deformation ones $(z \leftrightarrow \omega)$ was already analysed for the $(1+1) \mathrm{D}$ case in [73] and, in fact, the role of $z$ as a curvature also arises within the Drinfel'd-double approach, as commented in Section 3.1 (see $[29,30])$. Moreover, the "semidualization" approach in $2+$ 1 quantum gravity introduced in [59] (see also [60, 61]) provides a more complete Hopf algebraic understanding of this duality between the Planck scale $l_{p}$ and the cosmological constant $\omega=\rho^{2}=-\Lambda$ and shows its direct connection with the quantum version of the so-called Born reciprocity principle [74] between (now noncommutative) coordinates and (curved) momenta. This framework is also helpful in order to understand the existing constraints on contraction limits involving these two parameters that we will discuss in the sequel.

6.2. Contractions and the Planck Length. It has been shown (see [38] and references therein) that there exists a natural link between the deformed commutation relations (56) for the (A)dS algebras with $\omega \neq 0$ and $2+1$ quantum gravity. In the latter framework, the curvature and deformation parameters can be identified with the cosmological constant $\Lambda \equiv-\omega$ and the fundamental Planck length $l_{p} \equiv z$. Hence, by considering these identifications, the above results show that both limits $\Lambda \rightarrow 0$ and $l_{p} \rightarrow 0$ can be taken independently (if $\Lambda \rightarrow 0$ we obtain $\kappa$-Poincaré with $l_{p} \equiv z$ ) and that a "duality" $l_{p} \leftrightarrow \Lambda$ might exist. Nevertheless, in [38] it is emphasized that the contraction to $\kappa$-Poincare should be taken as the simultaneous limits $z \rightarrow 0$ and $\Lambda l_{p}^{2} \rightarrow 0$, due to the coupling $z=\sqrt{\Lambda} l_{p}$ existing in $2+1$ quantum gravity. This fact can be explained from a Lie bialgebra contraction approach [68] as follows.

Alternatively to the expressions (55) and (56) defining $U_{z}\left(s o_{\omega}(2,2)\right)$ that unify the Hopf structure for the quantum (A)dS and Poincaré algebras parametrized by $\omega$, one could have started from the quantum AdS algebra $U_{z}(s o(2,2))$ with $\omega=1\left(\right.$ or from $U_{z}(s o(3,1))$ with $\left.\omega=-1\right)$. Next the Lie bialgebra contraction analysis of the $r$-matrix (26) and Lie bialgebra (27) shows that there exists a unique quantum Inönü-Wigner contraction (a coboundary Lie bialgebra contraction) that ensures the convergence of both (26) and (27). This is defined through the new generators $Y^{\prime}$ and deformation parameter $z^{\prime}$ given by $[19,68]$

$$
\begin{aligned}
P_{0}^{\prime} & =\sqrt{\omega} P_{0}, \\
P_{i}^{\prime} & =\sqrt{\omega} P_{i}, \\
K_{i}^{\prime} & =K_{i}, \\
J^{\prime} & =J, \\
z^{\prime} & =\frac{z}{\sqrt{\omega}}
\end{aligned}
$$


( $\sqrt{\omega}=\rho$ plays the role of the usual Inönü-Wigner contraction parameter). This map is associated with the $\mathbb{Z}_{2}$-grading of $U_{z}(s o(2,2))$ given by the composition of the deformed parity and time-reversal (32):

$$
\Pi_{z} \Theta_{z}:\left(P_{0}, \mathbf{P}, \mathbf{K}, J ; z\right) \longrightarrow\left(-P_{0},-\mathbf{P}, \mathbf{K}, J ;-z\right) .
$$

By computing the coproduct and commutation relations for the new generators $Y^{\prime}$ from $U_{z}(s o(2,2))$ and then by applying the limit $\sqrt{\omega} \rightarrow 0$, one finds the $\kappa$-Poincaré algebra $U_{z^{\prime}}(i s o(2,1))$ in terms of the transformed deformation parameter $z^{\prime}$. Therefore, if $z^{\prime}$ is now taken as the Planck length $l_{p}$ and $\omega \equiv-\Lambda>0$, the map (58) means that $z=\sqrt{-\Lambda} l_{p}$, so that the contraction $\sqrt{-\Lambda} \rightarrow 0$ conveys the limit $z \rightarrow 0$ as well, leaving $z^{\prime} \equiv l_{p}$ as the only fundamental scale in $\kappa$ Poincaré, in agreement with [38].

6.3. "Bicrossproduct-Type" Basis. So far, most of the applications of the $\kappa$-Poincaré algebra have been developed by working in the so-called bicrossproduct basis [16, 22], in which the Lorentz sector has nondeformed commutation rules. Thus it is natural to wonder about the existence of a nonzero curvature counterpart of such a basis. This can be achieved by means of the $\omega$-generalization of the invertible nonlinear map introduced for $\kappa$-Poincaré in [16]

$$
\begin{aligned}
\widetilde{P}_{0} & =P_{0}, \\
\widetilde{J}=J & \\
\widetilde{P}_{i} & =e^{-(z / 2) P_{0}}\left\{\cosh \left(\frac{z}{2} \rho J\right) P_{i}-\rho \sinh \left(\frac{z}{2} \rho J\right) \epsilon_{i j} K_{j}\right\}, \\
\widetilde{K}_{i} & =e^{-(z / 2) P_{0}}\left\{\cosh \left(\frac{z}{2} \rho J\right) K_{i}\right. \\
& \left.+\frac{\sinh ((z / 2) \rho J)}{\rho} \epsilon_{i j} P_{j}\right\} .
\end{aligned}
$$

This transformation can be written as the following matrix transformation of $\mathbf{P}$ and $\mathbf{K}$ depending on functions of the generators of the isotropy subgroup of a worldline:

$$
\begin{aligned}
\left(\begin{array}{c}
\widetilde{P}_{1} \\
\widetilde{P}_{2} \\
\widetilde{K}_{1} \\
\widetilde{K}_{2}
\end{array}\right)= & \exp \left\{-\frac{z}{2} P_{0}\left(\begin{array}{llll}
1 & 0 & 0 & 0 \\
0 & 1 & 0 & 0 \\
0 & 0 & 1 & 0 \\
0 & 0 & 0 & 1
\end{array}\right)\right\} \\
& \cdot \exp \left\{-\frac{z}{2} J\left(\begin{array}{cccc}
0 & 0 & 0 & w \\
0 & 0 & -w & 0 \\
0 & -1 & 0 & 0 \\
1 & 0 & 0 & 0
\end{array}\right)\right\} \\
& \cdot\left(\begin{array}{c}
P_{1} \\
P_{2} \\
K_{1} \\
K_{2}
\end{array}\right) .
\end{aligned}
$$

The transformed coproduct and commutation rules of $U_{z}\left(s o_{\omega}(2,2)\right)$ in this new basis turn out to be

$$
\begin{aligned}
& \Delta\left(\widetilde{P}_{0}\right)=1 \otimes \widetilde{P}_{0}+\widetilde{P}_{0} \otimes 1, \\
& \Delta(\widetilde{J})=1 \otimes \widetilde{J}+\widetilde{J} \otimes 1, \\
& \Delta\left(\widetilde{P}_{i}\right)=e^{-z \widetilde{P}_{0}} \otimes \widetilde{P}_{i}+\widetilde{P}_{i} \otimes \cosh (z \rho \widetilde{J})-\rho \epsilon_{i j} \widetilde{K}_{j} \\
& \otimes \sinh (z \rho \widetilde{J}), \\
& \Delta\left(\widetilde{K}_{i}\right)=e^{-z \widetilde{P}_{0}} \otimes \widetilde{K}_{i}+\widetilde{K}_{i} \otimes \cosh (z \rho \widetilde{J})+\epsilon_{i j} \widetilde{P}_{j} \\
& \otimes \frac{\sinh (z \rho \widetilde{J})}{\rho}, \\
& {\left[\widetilde{J}, \widetilde{P}_{i}\right]=\epsilon_{i j} \widetilde{P}_{j} \text {, }} \\
& {\left[\widetilde{J}, \widetilde{K}_{i}\right]=\epsilon_{i j} \widetilde{K}_{j} \text {, }} \\
& {\left[\widetilde{J}, \widetilde{P}_{0}\right]=0 \text {, }} \\
& {\left[\widetilde{P}_{0}, \widetilde{K}_{i}\right]=-\widetilde{P}_{i},} \\
& {\left[\widetilde{P}_{0}, \widetilde{P}_{i}\right]=\omega \widetilde{K}_{i},} \\
& {\left[\widetilde{P}_{1}, \widetilde{P}_{2}\right]=-\omega \frac{\sinh (2 z \rho \widetilde{J})}{2 z \rho},} \\
& {\left[\widetilde{K}_{1}, \widetilde{K}_{2}\right]=-\frac{\sinh (2 z \rho \widetilde{J})}{2 z \rho},} \\
& {\left[\widetilde{P}_{i}, \widetilde{K}_{j}\right]=\delta_{i j}\left\{\frac{e^{-2 z \widetilde{P}_{0}}-\cosh (2 z \rho \widetilde{J})}{2 z}\right.} \\
& \left.-\frac{\tan (z \rho)}{2 \rho}\left(\widetilde{\mathbf{P}}^{2}+\omega \widetilde{\mathbf{K}}^{2}\right)\right\}+\frac{\tan (z \rho)}{\rho}\left(\widetilde{P}_{j} \widetilde{P}_{i}\right. \\
& \left.+\omega \widetilde{K}_{i} \widetilde{K}_{j}\right)
\end{aligned}
$$

Therefore, the Lorentz sector remains deformed in the quantum (A)dS algebras with $\omega \neq 0$, while the contraction $\omega=0$ $(\rho \rightarrow 0)$ produces the $\kappa$-Poincare algebra in the bicrossproduct basis, in which the only deformed commutation rules are $\left[\widetilde{P}_{i}, \widetilde{K}_{j}\right]$. However, although the Lorentz sector in $\kappa$-Poincaré is nondeformed, the quantum deformation is still kept in the coproduct for the boost generators (62). Note also that this new coproduct is not invariant under the map $\sigma \circ \mathscr{T}$.

The corresponding deformed Casimirs are obtained from (57) and read

$$
\begin{aligned}
\mathscr{C} & =4 \cos (z \rho)\left\{\frac{\sinh ^{2}\left((z / 2) \widetilde{P}_{0}\right)}{z^{2}} \cosh ^{2}\left(\frac{z}{2} \rho \widetilde{J}\right)\right. \\
& \left.+\frac{\sinh ^{2}((z / 2) \rho \widetilde{J})}{z^{2}} \cosh ^{2}\left(\frac{z}{2} \widetilde{P}_{0}\right)\right\}-\frac{\sin (z \rho)}{z \rho}
\end{aligned}
$$




$$
\begin{aligned}
& \cdot e^{z \widetilde{P}_{0}}\left\{\cosh (z \rho \widetilde{J})\left(\widetilde{\mathbf{P}}^{2}+\omega \widetilde{\mathbf{K}}^{2}\right)-2 \rho\right. \\
& \left.\cdot \sinh (z \rho \widetilde{J})\left(\widetilde{K}_{1} \widetilde{P}_{2}-\widetilde{K}_{2} \widetilde{P}_{1}\right)\right\}, \\
& \mathscr{W}=-\cos (z \rho) \frac{\sinh (z \rho \widetilde{J})}{z \rho} \frac{\sinh \left(z \widetilde{P}_{0}\right)}{z}+\frac{\sin (z \rho)}{z \rho} \\
& \cdot e^{z \widetilde{P}_{0}}\left\{\cosh (z \rho \widetilde{J})\left(\widetilde{K}_{1} \widetilde{P}_{2}-\widetilde{K}_{2} \widetilde{P}_{1}\right)\right. \\
& \left.-\frac{\sinh (z \rho \widetilde{J})}{2 \rho}\left(\widetilde{\mathbf{P}}^{2}+\omega \widetilde{\mathbf{K}}^{2}\right)\right\} .
\end{aligned}
$$

We remark that for both bases the full Hopf structure $U_{z}\left(s o_{\omega}(2,2)\right)$ is invariant under the quantum involutions (32) (as it should be) and also under the "classical" symmetry

$$
\left(P_{0}, P_{1}, P_{2}, K_{1}, K_{2}, J\right) \longrightarrow\left(P_{0}, P_{2}, P_{1}, K_{2}, K_{1},-J\right),
$$

which shows that the equivalences $P_{1} \leftrightarrow P_{2}$ and $K_{1} \leftrightarrow K_{2}$ are preserved by this quantum deformation. This, in turn, means that there is no privileged space/boost direction. The analogous (dual) maps to (65) in the noncommutative spacetimes and spaces of worldlines are given by the interchange $\widehat{s}_{1} \leftrightarrow \widehat{s}_{2}$ and (53), respectively.

\section{Concluding Remarks}

We have presented a unified and global study of the $\kappa$-deformation of the (A)dS and Poincaré algebras and groups in $2+1$ dimensions by making use of an explicit contraction parameter $\omega$ that corresponds to the curvature/cosmological constant of the underlying classical spacetimes. We remark that the limit $\omega \rightarrow 0$ is always well defined in all the expressions, thus providing a straightforward Poincaré/Minkowskian counterpart of all the results here presented.

At the quantum algebra level, we have introduced a new basis (60) for the quantum (A)dS algebras, which is the analogous of the $\kappa$-Poincaré bicrossproduct basis. As far as the (dual) quantum groups are concerned, the results cover the noncommutative (A)dS spacetimes (49) up to secondorder in the deformation parameter, thus generalizing the $\kappa$-Minkowskian spacetime (33) which turns out to be their common first-order seed. Furthermore, we have presented the first approach, to the best of our knowledge, to noncommutative spaces of worldlines. For the three relativistic cases they are related to phase spaces $(\widehat{\mathbf{x}}, \widehat{\xi}) \leftrightarrow(\mathbf{P}, \mathbf{K})$, which are different from the $\kappa$-Poincaré phase spaces proposed in $[29,30]$ (see also [75]). We also remark that an appropriate treatment of the classical (nondeformed) structures have allowed us to write both noncommutative spacetimes and spaces of worldlines in terms of ambient ("global") coordinates, by starting with the particular expressions (16) and (23) for the ("local") parametrizations of such spaces. In such ambient coordinates, the noncommutative spaces turn out to be determined through quadratic commutation relations (50) and (52). Moreover, they are shown to be symmetric with respect to some maps that generalize the $P_{1} \leftrightarrow P_{2}$ and $K_{1} \leftrightarrow K_{2}$ invariance of the dual quantum algebras.

It is worth stressing that the connection between the Poisson-Lie group approach presented here and the role that classical $r$-matrices and Drinfel'd-doubles play in the context of $2+1$ quantum gravity [76-84] has been studied in detail in the works [85-89]. Also, the deformed Casimir operators (57) (or (64)) can be used to provide modified dispersion relations, which should be related to those appearing in several phenomenological approaches to quantum gravity (see [90-93]).

Some comments on other possible quantum deformations of the (A)dS algebras are in order. Since any possible quantum deformation of the (A)dS algebras has to come from a classical $r$-matrix, a complete classification of the latter would be certainly useful in order to obtain and analyse other physically interesting quantum (A)dS groups (for each of them, the vector fields displayed in Table 2 would give rise to the associated Poisson noncommutative spaces). In this respect, the full classification of classical $r$-matrices for the $(3+1)$ D Poincaré Lie algebra can be found in [94]. For the (2 $+1) \mathrm{D}(\mathrm{A}) \mathrm{d} S$ algebras a similar result has been recently given in [95] (see also [96]).

On the other hand, the generalization of the $(2+1) \mathrm{D} \kappa$ (A)dS algebras and groups to the $(3+1) \mathrm{D}$ case can be obtained by generalizing the classical $r$-matrix (26) to (see [68])

$$
r=z\left(K_{1} \wedge P_{1}+K_{2} \wedge P_{2}+K_{3} \wedge P_{3}\right)+z \sqrt{\omega} J_{1} \wedge J_{2},
$$

which include a term $J_{1} \wedge J_{2}$ coming from the rotation sector. This term does not appear either in the $(3+1) \mathrm{D}$ $\kappa$-Poincaré algebra (with $\omega=0$ ) or in the $(2+1) \mathrm{D} \kappa$ (A)dS algebras. A twisted version of (66) with a second deformation parameter $\vartheta$ was considered in [97] by imposing some physical requirements, and the very same classical $r$ matrix has been derived in [98] from a Drinfeld-double approach; namely,

$$
\begin{aligned}
r= & z\left(K_{1} \wedge P_{1}+K_{2} \wedge P_{2}+K_{3} \wedge P_{3}\right)+z \sqrt{\omega} J_{1} \wedge J_{2} \\
& +\vartheta J_{3} \wedge P_{0} .
\end{aligned}
$$

The corresponding Poisson-Hopf algebra has been recently constructed in [99], so obtaining the $(3+1) \mathrm{D}$ Poisson version of the coproduct (62), commutation relations (63), and Casimirs (64). The associated $(3+1) \mathrm{D}$ noncommutative spacetime, generalizing (49), is currently under investigation.

Finally, we recall that other different quantum (A)dS deformations have been obtained by working in a conformal basis instead of a pure kinematical one, for instance, $s o(3,2)$ $[100,101]$ and $s o(4,2)[47,102-104]$, and the Drinfel'd-Jimbo quantum AdS space at roots of unity has been worked out in [105]. Also, other fuzzy $[106,107]$ and covariant [44] noncommutative (A)dS spacetimes have also been constructed.

\section{Conflicts of Interest}

The authors declare that they have no conflicts of interest. 


\section{Acknowledgments}

This work has been partially supported by Ministerio de Economía y Competitividad (MINECO, Spain) under Grants MTM2013-43820-P and MTM2016-79639-P (AEI/FEDER, UE), by Junta de Castilla y León (Spain) under Grants BU278U14 and VA057U16, and by the Action MP1405 QSPACE from the European Cooperation in Science and Technology (COST).

\section{References}

[1] S. Majid, "Hopf algebras for physics at the Planck scale," Classical and Quantum Gravity, vol. 5, no. 12, pp. 1587-1606, 1988.

[2] P. P. Kulish and N. Yu. Reshetikhin, "Quantum linear problem for the sine-Gordon equation and higher representations," Proceedings of the Steklov Institute of Mathematics, vol. 101, pp. 101-110, 1981 (Russian), English translation: Journal of Soviet Mathematics, vol. 23, no. 4, pp. 2435-2441, 1983.

[3] V. G. Drinfeld, "Quantum groups," in Proceedings of the International Congress of Mathematicians, A. V. Gleason, Ed., vol. 1, pp. 798-820, AMS, Berkeley, Calif, USA, 1987.

[4] M. Jimbo, "Aq-difference analogue of $\mathrm{U}(\mathrm{g})$ and the Yang-Baxter equation," Letters in Mathematical Physics, vol. 10, no. 1, pp. 6369, 1985.

[5] L. A. Takhtajan, "Lectures on quantum groups", in Introduction to Quantum Group and Integrable Massive Models of Quantum Field Theory (Nankai, 1989), Nankai Lectures Math. Phys., pp. 69-197, World Scientific, River Edge, NJ, USA, 1990.

[6] N. Yu. Reshetikhin, L. A. Takhtadzhyan, and L. D. Faddeev, "Quantization of Lie groups and Lie algebras," Algebra i Analiz, vol. 1, no. 1, pp. 178-206, 1989 (Russian), English translation: Leningrad Mathematical Journal, vol. 1, no. 1, pp. 193-225, 1990.

[7] V. Chari and A. Pressley, A Guide to Quantum Groups, Cambridge University Press, Cambridge, UK, 1994.

[8] S. Majid, Foundations of Quantum Group Theory, Cambridge University Press, Cambridge, UK, 1995.

[9] J. Lukierski, H. Ruegg, A. Nowicki, and V. N. Tolstoy, " $q$ deformation of Poincaré algebra," Physics Letters. B. Particle Physics, Nuclear Physics and Cosmology, vol. 264, no. 3-4, pp. 331-338, 1991.

[10] E. Celeghini, R. Giachetti, E. Sorace, and M. Tarlini, "The quantum Heisenberg group $H(1)_{q}$," Journal of Mathematical Physics, vol. 32, no. 5, pp. 1155-1158, 1991.

[11] E. Celeghini, R. Giachetti, E. Sorace, and M. Tarlini, “The threedimensional Euclidean quantum group $E(3)_{q}$ and its $R$-matrix," Journal of Mathematical Physics, vol. 32, no. 5, pp. 1159-1165, 1991.

[12] F. Bonechi, E. Celeghini, R. Giachetti, E. Sorace, and M. Tarlini, "Inhomogeneous quantum groups as symmetries of phonons," Physical Review Letters, vol. 68, no. 25, pp. 3718-3720, 1992.

[13] S. Giller, P. Kosinski, M. Majewski, P. Maslanka, and J. Kunz, "More about the q-deformed Poincaré algebra," Physics Letters. B. Particle Physics, Nuclear Physics and Cosmology, vol. 286, no. 1-2, pp. 57-62, 1992.

[14] J. Lukierski, A. Nowicki, and H. Ruegg, "New quantum Poincaré algebra and $k$-deformed field theory," Physics Letters. B. Particle Physics, Nuclear Physics and Cosmology, vol. 293, no. 3-4, pp. 344-352, 1992.
[15] P. Maslanka, "The $n$-dimensional kappa -Poincaré algebra and group," Journal of Physics. A. Mathematical and General, vol. 26, no. 24, pp. L1251-L1253, 1993.

[16] S. Majid and H. Ruegg, "Bicrossproduct structure of $k$-Poincaré group and non-commutative geometry," Physics Letters. B. Particle Physics, Nuclear Physics and Cosmology, vol. 334, no. 34, pp. 348-354, 1994.

[17] S. Zakrzewski, "Quantum Poincaré group related to the kappa -Poincaré algebra," Journal of Physics. A. Mathematical and General, vol. 27, no. 6, pp. 2075-2082, 1994.

[18] J. Lukierski and H. Ruegg, "Quantum $\kappa$-Poincaré in any dimension," Physics Letters B, vol. 329, no. 2-3, pp. 189-194, 1994.

[19] A. Ballesteros, F. J. Herranz, M. A. del Olmo, and M. Santander, "Quantum $(2+1)$ kinematical algebras: a global approach," Journal of Physics. A. Mathematical and General, vol. 27, no. 4, pp. 1283-1297, 1994.

[20] A. Ballesteros, F. J. Herranz, M. A. del Olmo, and M. Santander, "Four-dimensional quantum affine algebras and space-time qsymmetries," Journal of Mathematical Physics, vol. 35, no. 9, pp. 4928-4940, 1994.

[21] A. Ballesteros, F. J. Herranz, M. A. del Olmo, and M. Santander, "A new "null-plane" quantum Poincaré algebra," Physics Letters. B. Particle Physics, Nuclear Physics and Cosmology, vol. 351, no. 1-3, pp. 137-145, 1995.

[22] J. Lukierski, H. Ruegg, and W. J. Zakrzewski, "Classical and quantum mechanics of free $\kappa$-relativistic systems," Annals of Physics, vol. 243, no. 1, pp. 90-116, 1995.

[23] A. Ballesteros, F. J. Herranz, M. A. del Olmo, C. M. Pereña, and M. Santander, "Non-standard quantum $(1+1)$ Poincaré group: a T-matrix approach," Journal of Physics. A. Mathematical and General, vol. 28, no. 24, pp. 7113-7125, 1995.

[24] G. Amelino-Camelia, "Testable scenario for relativity with minimum length," Physics Letters B, vol. 510, no. 1-4, pp. 255$263,2001$.

[25] G. Amelino-Camelia, "Relativity in spacetimes with shortdistance structure governed by an observer-independent (Planckian) length scale," International Journal of Modern Physics D, vol. 11, no. 1, pp. 35-59, 2002.

[26] G. Amelino-Camelia, "Doubly-special relativity: first results and key open problems," International Journal of Modern Physics D, vol. 11, no. 10, pp. 1643-1669, 2002.

[27] J. Magueijo and L. Smolin, "Lorentz invariance with an invariant energy scale," Physical Review Letters, vol. 88, Article ID 190403, 2002.

[28] J. Kowalski-Glikman and S. Nowak, "Doubly special relativity theories as different bases of $k$-Poincaré algebra," Physics Letters. B. Particle Physics, Nuclear Physics and Cosmology, vol. 539, no. 1-2, pp. 126-132, 2002.

[29] J. Kowalski-Glikman, "De Sitter space as an arena for doubly special relativity," Physics Letters B, vol. 547, no. 3-4, pp. 291-296, 2002.

[30] J. Kowalski-Glikman and S. Nowak, "Doubly special relativity and de Sitter space," Classical and Quantum Gravity, vol. 20, no. 22, pp. 4799-4816, 2003.

[31] J. Lukierski and A. Nowicki, "Doubly special relativity versus $\kappa$ deformation of relativistic kinematics," International Journal of Modern Physics A, vol. 18, no. 1, pp. 7-18, 2003.

[32] Á. Ballesteros, N. Rossano Bruno, and F. J. Herranz, "A new 'doubly special relativity' theory from a quantum Weyl-Poincaré algebra," Journal of Physics. A. Mathematical and General, vol. 36, no. 42, pp. 10493-10503, 2003. 
[33] J. Polchinski, “TASI lectures on D-branes," in Proceedings of the TASI 96: Fields, Strings, and Duality, C. Efthimiou and B. Greene, Eds., pp. 293-356, World Scientific, Singapore, 1997, http://xxx.lanl.gov/abs/hep-th/9611050.

[34] S. Carlip, "Quantum gravity: a progress report," Reports on Progress in Physics, vol. 64, no. 8, pp. 885-942, 2001.

[35] L. Smolin, "Quantum gravity with a positive cosmological constant," https://arxiv.org/abs/hep-th/0209079, In press.

[36] S. Förste, "Strings, branes and extra dimensions," Fortschritte der Physik. Progress of Physics, vol. 50, no. 3-4, pp. 221-403, 2002.

[37] L. Freidel, E. R. Livine, and C. Rovelli, "Spectra of length and area in $(2+1)$ Lorentzian loop quantum gravity," Classical and Quantum Gravity, vol. 20, no. 8, pp. 1463-1478, 2003.

[38] G. Amelino-Camelia, L. Smolin, and A. Starodubtsev, "Quantum symmetry, the cosmological constant and Planck-scale phenomenology," Classical and Quantum Gravity, vol. 21, no. 13, pp. 3095-3110, 2004.

[39] L. Freidel, J. Kowalski-Glikman, and L. Smolin, " $2+1$ gravity and doubly special relativity," Physical Review. D. Third Series, vol. 69, no. 4, Article ID 044001, 7 pages, 2004.

[40] H. S. Snyder, "Quantized space-time," Physical Review, vol. 71, no. 1, pp. 38-41, 1947.

[41] P. Podleś and S. L. Woronowicz, "Quantum deformation of Lorentz group," Communications in Mathematical Physics, vol. 130, no. 2, pp. 381-431, 1990.

[42] S. Doplicher, K. Fredenhagen, and J. E. Roberts, "The quantum structure of spacetime at the Planck scale and quantum fields," Communications in Mathematical Physics, vol. 172, no. 1, pp. 187220, 1995.

[43] R. J. Szabo, "Quantum field theory on noncommutative spaces," Physics Reports A: Review Section of Physics Letters, vol. 378, no. 4, pp. 207-299, 2003.

[44] J. J. Heckman and H. Verlinde, "Covariant non-commutative space-time," Nuclear Physics. B. Theoretical, Phenomenological, and Experimental High Energy Physics. Quantum Field Theory and Statistical Systems, vol. 894, pp. 58-74, 2015.

[45] M. Maggiore, "The algebraic structure of the generalized uncertainty principle," Physics Letters B, vol. 319, no. 1-3, pp. 83-86, 1993.

[46] L. J. Garay, "Quantum gravity and minimum length," International Journal of Modern Physics A, vol. 10, no. 2, pp. 145-165, 1995.

[47] A. Ballesteros, N. R. Bruno, and F. J. Herranz, "A noncommutative Minkowskian spacetime from a quantum AdS algebra," Physics Letters. B. Particle Physics, Nuclear Physics and Cosmology, vol. 574, no. 3-4, pp. 276-282, 2003.

[48] A. Marcianò, G. Amelino-Camelia, N. R. Bruno, G. Gubitosi, G. Mandanici, and A. Melchiorri, "Interplay between curvature and Planck-scale effects in astrophysics and cosmology," Journal of Cosmology and Astroparticle Physics, vol. 2010, no. 06, p. 030, 2010.

[49] G. Amelino-Camelia, L. Barcaroli, DG. Amico, N. Loret, and G. Rosati, "IceCube and GRB neutrinos propagating in quantum spacetime," Physics Letters B, vol. 761, pp. 318-325, 2016.

[50] G. Amelino-Camelia, G. Gubitosi, and G. Palmisano, "Pathways to relativistic curved momentum spaces: de Sitter case study," International Journal of Modern Physics. D. Gravitation, Astrophysics, Cosmology, vol. 25, no. 2, Article ID 1650027, 36 pages, 2016.

[51] L. Barcaroli and G. Gubitosi, "Kinematics of particles with quantum-de Sitter-inspired symmetries," Physical Review D, vol. 93, no. 12, Article ID 124063, 2016.
[52] A. Ballesteros, F. J. Herranz, O. Ragnisco, and M. Santander, "Contractions, deformations and curvature," International Journal of Theoretical Physics, vol. 47, no. 3, pp. 649-663, 2008.

[53] F. J. Herranz and M. Santander, "(Anti)de Sitter/Poincaré symmetries and representations from Poincaré/Galilei through a classical deformation approach," Journal of Physics. A. Mathematical and Theoretical, vol. 41, no. 1, Article ID 015204, p. 16, 2008.

[54] V. G. Drinfel'd, "On Poisson homogeneous spaces of PoissonLie groups," Theoretical and Mathematical Physics, vol. 95, no. 2, pp. 524-525, 1993.

[55] M. S. Dijkhuizen and T. H. Koornwinder, "Quantum homogeneous spaces, duality and quantum 2-spheres," Geometriae Dedicata, vol. 52, no. 3, pp. 291-315, 1994.

[56] S. Zakrzewszki, "Poisson homogeneous spaces," in Quantum Groups, Formalism and Applications, J. Lukierski, Z. Popowicz, and J. Sobczyk, Eds., pp. 629-639, PWN, Warszaw, Poland, 1995.

[57] A. G. Reyman, "Poisson structures related to quantum groups," in Quantum Groups and Their Applications in Physics, L. Castellani and J. Wess, Eds., pp. 407-444, IOS Press, Amsterdam, The Netherlands, 1996.

[58] N. Ciccoli, "Quantum planes and quantum cylinders from Poisson homogeneous spaces," Journal of Physics. A. Mathematical and General, vol. 29, no. 7, pp. 1487-1495, 1996.

[59] S. Majid and B. J. Schroers, " $q$-deformation and semidualization in 3D quantum gravity," Journal of Physics. A. Mathematical and Theoretical, vol. 42, no. 42, Article ID 425402, p. 40, 2009.

[60] P. K. Osei and B. J. Schroers, "On the semiduals of local isometry groups in three-dimensional gravity," Journal of Mathematical Physics, vol. 53, no. 7, Article ID 073510, p. 26, 2012.

[61] P. K. Osei and B. J. Schroers, "Classical $r$-matrices via semidualisation," Journal of Mathematical Physics, vol. 54, no. 10, Article ID 101702, p. 17, 2013.

[62] E. Inonu and E. P. Wigner, "On the contraction of groups and their representations," Proceedings of the National Academy of Sciences of the United States of America, vol. 39, no. 6, pp. 510524, 1953.

[63] H. Bacry and J.-M. Lévy-Leblond, "Possible kinematics," Journal of Mathematical Physics, vol. 9, pp. 1605-1614, 1968.

[64] F. J. Herranz and M. Santander, "The general solution of the real $\mathbb{Z}_{2}^{\otimes N}$ graded contractions of $s o(N+1)$," Journal of Physics. A. Mathematical and General, vol. 29, no. 20, pp. 6643-6652, 1996.

[65] R. Gilmore, Lie Groups, Lie Algebras, and Some of Their Applications, Wiley, New York, NY, USA, 1994.

[66] F. J. Herranz and M. Santander, "Homogeneous phase spaces: the Cayley-Klein framework," in Geometria y Fisica, J. F. Cariñena, E. Martinez, and M. F. Rañada, Eds., vol. XXXII of Memorias de la Real Academia de Ciencias, pp. 59-84, Real Academia de Ciencias, Madrid, Spain, 1998, https://arxiv.org/abs/physics/9702030.

[67] F. J. Herranz and M. Santander, "Conformal symmetries of spacetimes," Journal of Physics. A. Mathematical and General, vol. 35, no. 31, pp. 6601-6618, 2002.

[68] A. Ballesteros, N. A. Gromov, F. J. Herranz, M. A. del Olmo, and M. Santander, "Lie bialgebra contractions and quantum deformations of quasi-orthogonal algebras," Journal of Mathematical Physics, vol. 36, no. 10, pp. 5916-5937, 1995.

[69] R. Aldrovandi, A. L. Barbosa, L. C. Crispino, and J. G. Pereira, "Non-relativistic spacetimes with cosmological constant," Classical and Quantum Gravity, vol. 16, no. 2, pp. 495-506, 1999. 
[70] V. G. Drinfel'd, "Hamiltonian structures on Lie groups, Lie bialgebras and the geometric meaning of classical Yang-Baxter equations," Soviet Mathematics Doklady, vol. 27, pp. 68-71, 1983.

[71] N. R. Bruno, G. Amelino-Camelia, and J. Kowalski-Glikman, "Deformed boost transformations that saturate at the Planck scale," Physics Letters, Section B: Nuclear, Elementary Particle and High-Energy Physics, vol. 522, no. 1-2, pp. 133-138, 2001.

[72] N. R. Bruno, "Group of boost and rotation transformations with two observer-independent scales," Physics Letters B, vol. 547, no. 1-2, pp. 109-115, 2002.

[73] A. Ballesteros, F. J. Herranz, M. A. del Olmo, and M. Santander, "Classical deformations, Poisson-Lie contractions, and quantization of dual Lie bialgebras," Journal of Mathematical Physics, vol. 36, no. 2, pp. 631-640, 1995.

[74] M. Born, "A suggestion for unifying quantum theory and relativity," Proceedings of the Royal Society of London A, vol. 165, pp. 291-303, 1938.

[75] A. Blaut, M. Daszkiewicz, J. Kowalski-Glikman, and S. Nowak, "Phase spaces of doubly special relativity," Physics Letters. B. Particle Physics, Nuclear Physics and Cosmology, vol. 582, no. 1-2, pp. 82-85, 2004.

[76] A. Y. Alekseev and A. Z. Malkin, "Symplectic structure of the moduli space of flat connection on a Riemann surface," Communications in Mathematical Physics, vol. 169, no. 1, pp. 99119, 1995.

[77] V. V. Fock and A. A. Rosly, "Poisson structure on moduli of flat connections on Riemann surfaces and r-matrix," ITEP-72-92, 1992.

[78] V. V. Fock and A. A. Rosly, "Poisson structure on moduli of flat connections on Riemann surfaces and $r$-matrix," American Mathematical Society Translations, vol. 191, pp. 67-86, 1999.

[79] A. Achúcarro and P. K. Townsend, "A Chern-Simons action for three-dimensional anti-de Sitter supergravity theories," Physics Letters B, vol. 180, no. 1-2, pp. 89-92, 1986.

[80] E. Witten, " $2+1$ dimensional gravity as an exactly soluble system," Nuclear Physics B, vol. 311, no. 1, pp. 46-78, 1988.

[81] C. Meusburger and B. J. Schroers, "Quaternionic and PoissonLie structures in three-dimensional gravity: the cosmological constant as deformation parameter," Journal of Mathematical Physics, vol. 49, no. 8, 083510, 27 pages, 2008.

[82] C. Meusburger and B. J. Schroers, "Generalised Chern-Simons actions for 3d gravity and $k$-Poincaré symmetry," Nuclear Physics. B. Theoretical, Phenomenological, and Experimental High Energy Physics. Quantum Field Theory and Statistical Systems, vol. 806, no. 3, pp. 462-488, 2009.

[83] G. Papageorgiou and B. Schroers, "A Chern-Simons approach to Galilean quantum gravity in $2+1$ dimensions," Journal of High Energy Physics, vol. 2009, no. 11, p. 009, 2009.

[84] G. Papageorgiou and B. J. Schroers, "Galilean quantum gravity with cosmological constant and the extended q-Heisenberg algebra," Journal of High Energy Physics, vol. 11, Article ID 020, 2010.

[85] A. Ballesteros, F. J. Herranz, and C. Meusburger, "Threedimensional gravity and Drinfel'd doubles: spacetimes and symmetries from quantum deformations," Physics Letters. B. Particle Physics, Nuclear Physics and Cosmology, vol. 687, no. 45, pp. 375-381, 2010.

[86] A. Ballesteros, F. J. Herranz, and C. Meusburger, "Drinfel'd doubles for (2+1)-gravity," Classical and Quantum Gravity, vol. 30, no. 15, Article ID 155012, p. 20, 2013.
[87] A. Ballesteros, F. J. Herranz, C. Meusburger, and P. Naranjo, "Twisted $(2+1) k$-AdS Algebra, Drinfel'd Doubles and NonCommutative Spacetimes," SIGMA. Symmetry, Integrability and Geometry. Methods and Applications, vol. 10, article 052, p. 26, 2014.

[88] A. Ballesteros, F. J. Herranz, and C. Meusburger, "A $(2+1)$ noncommutative Drinfel'd double spacetime with cosmological constant," Physics Letters. B. Particle Physics, Nuclear Physics and Cosmology, vol. 732, pp. 201-209, 2014.

[89] A. Ballesteros, C. Meusburger, and P. Naranjo, "AdS Poisson homogeneous spaces and Drinfel'd doubles," Journal of Physics A: Mathematical and Theoretical, vol. 50, no. 39, Article ID 395202, 26 pages, 2017.

[90] G. Amelino-Camelia, J. Ellis, N. E. Mavromatos, D. V. Nanopoulos, and S. Sarkar, "Tests of quantum gravity from observations of big gamma-ray bursts," Nature, vol. 393, pp. 763-765, 1998.

[91] G. Amelino-Camelia, "Doubly-special relativity: Facts, myths and some key open issues," Symmetry, vol. 2, no. 1, pp. 230-271, 2010.

[92] G. Amelino-Camelia, "Quantum-spacetime phenomenology," Living Reviews in Relativity, vol. 16, article 5, 2013.

[93] D. Mattingly, "Modern tests of Lorentz invariance," Living Reviews in Relativity, vol. 8, no. 1, Article ID 5, 84 pages, 2005.

[94] S. Zakrzewski, "Poisson structures on Poincaré group," Communications in Mathematical Physics, vol. 185, no. 2, pp. 285-311, 1997.

[95] A. Borowiec, J. Lukierski, and V. N. Tolstoy, "Quantum deformations of $D=4$ Euclidean, Lorentz, Kleinian and quaternionic $\mathrm{o}^{\star}(4)$ symmetries in unified $\mathrm{o}(4 ; \mathrm{C})$ setting," Physics Letters, Section B: Nuclear, Elementary Particle and High-Energy Physics, vol. 754, pp. 176-181, 2016.

[96] A. Ballesteros, F. J. Herranz, and F. Musso, "On quantum deformations of (anti-)de Sitter algebras in $(2+1)$ dimensions," Journal of Physics: Conference Series, vol. 532, Article ID 012002, 12 pages, 2014.

[97] F. J. Herranz, A. Ballesteros, and N. R. Bruno, "On $3+1$ anti-de Sitter and de Sitter Lie bialgebras with dimensionful deformation parameters," Czechoslovak Journal of Physics, vol. 54, no. 11, pp. 1321-1327, 2004.

[98] A. Ballesteros, F. J. Herranz, and P. Naranjo, “Towards $(3+1)$ gravity through Drinfel'd doubles with cosmological constant," Physics Letters. B. Particle Physics, Nuclear Physics and Cosmology, vol. 746, pp. 37-43, 2015.

[99] Á. Ballesteros, F. J. Herranz, F. Musso, and P. Naranjo, "The $\kappa$ (A)dS quantum algebra in $(3+1)$ dimensions," Physics Letters, Section B: Nuclear, Elementary Particle and High-Energy Physics, vol. 766, pp. 205-211, 2017.

[100] F. J. Herranz, "Non-standard quantum so $(3,2)$ and its contractions," Journal of Physics. A. Mathematical and General, vol. 30, no. 17, pp. 6123-6129, 1997.

[101] J. Lukierski, V. D. Lyakhovsky, and M. Mozrzymas, " $k$ deformations of $D=3$ conformal versus deformations of $D=4$ AdS symmetries," Modern Physics Letters A. Particles and Fields, Gravitation, Cosmology, Nuclear Physics, vol. 18, no. 11, pp. 753769, 2003.

[102] J. Lukierski, V. Lyakhovsky, and M. Mozrzymas, " $k$ deformations of $D=4$ Weyl and conformal symmetries," Physics Letters. B. Particle Physics, Nuclear Physics and Cosmology, vol. 538, no. 3-4, pp. 375-384, 2002.

[103] N. Aizawa, F. J. Herranz, J. Negro, and M. . del Olmo, “Twisted conformal algebra so $(4,2)$," Journal of Physics. A. Mathematical and General, vol. 35, no. 39, pp. 8179-8196, 2002. 
[104] F. J. Herranz, "New quantum conformal algebras and discrete symmetries," Physics Letters. B. Particle Physics, Nuclear Physics and Cosmology, vol. 543, no. 1-2, pp. 89-97, 2002.

[105] H. Steinacker, "Quantum anti-de Sitter space and sphere at roots of unity," Advances in Theoretical and Mathematical Physics, vol. 4, no. 1, pp. 155-208, 2000.

[106] D. Jurman and H. Steinacker, "2D fuzzy anti-de Sitter space from matrix models," Journal of High Energy Physics, vol. 2014, no. 1, article no. 100, 2014.

[107] M. Burić and J. Madore, "Noncommutative de Sitter and FRW spaces," The European Physical Journal C, vol. 75, no. 10, Article ID 502, 11 pages, 2015. 

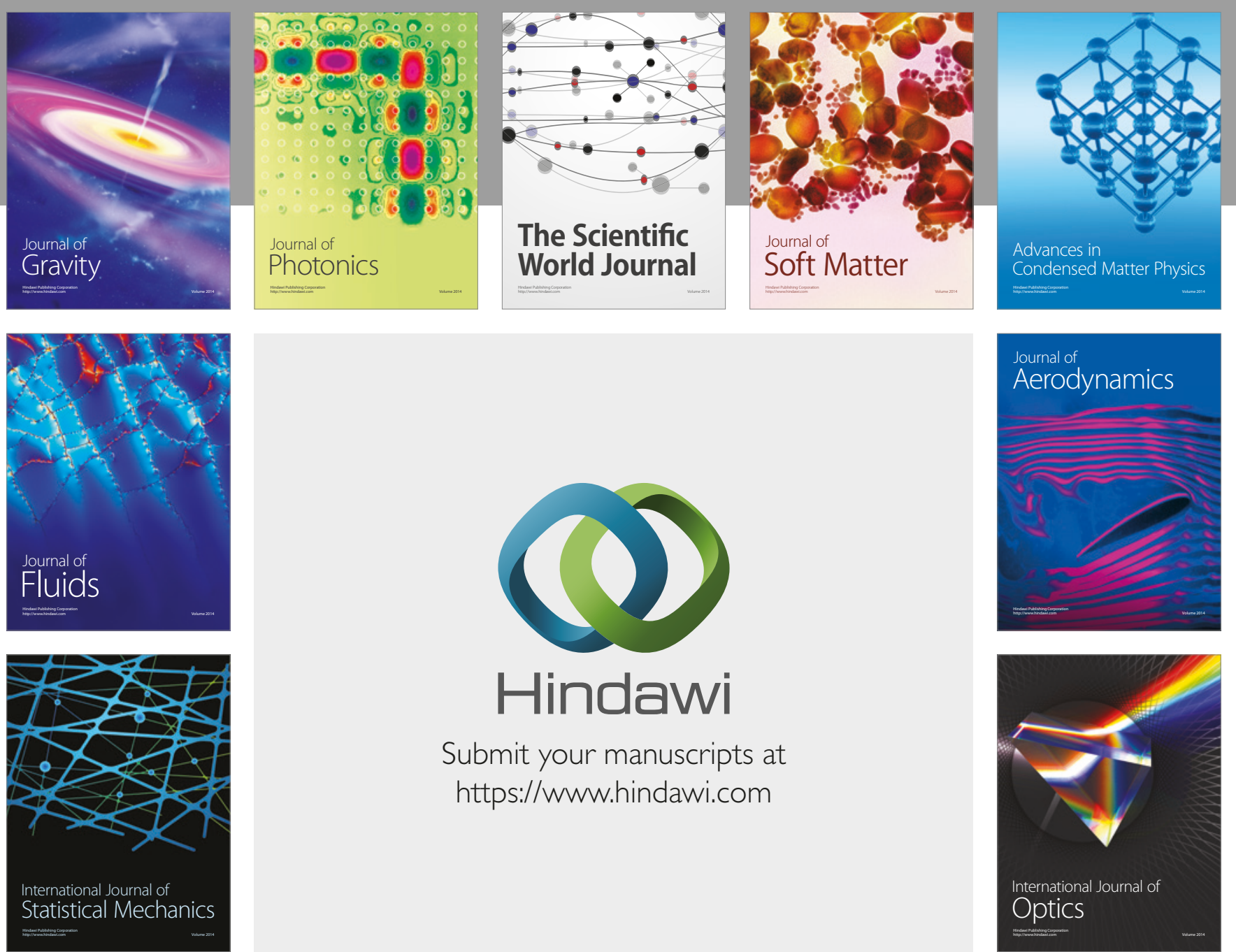

Submit your manuscripts at

https://www.hindawi.com
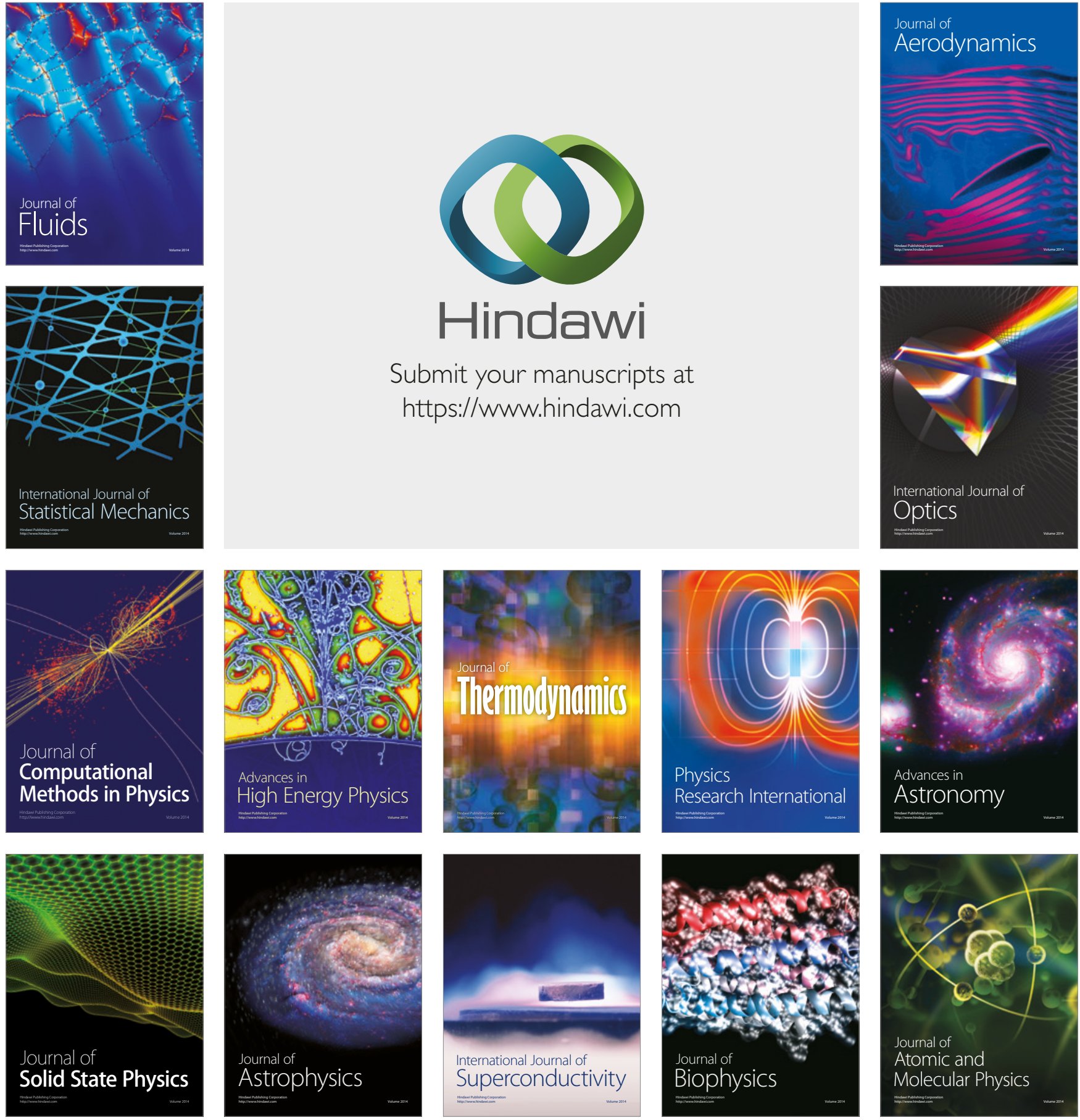